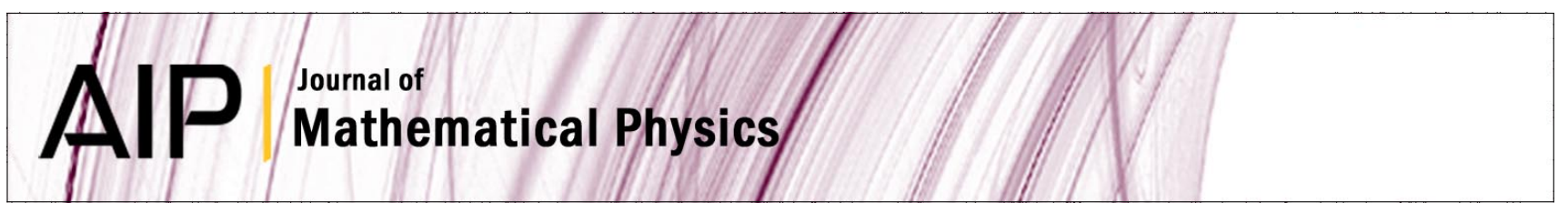

\title{
Translation-covariant Markovian master equation for a test particle in a quantum fluid
}

\author{
Bassano Vacchini
}

Citation: J. Math. Phys. 42, 4291 (2001); doi: 10.1063/1.1386409

View online: http://dx.doi.org/10.1063/1.1386409

View Table of Contents: http://jmp.aip.org/resource/1/JMAPAQ/v42/i9

Published by the American Institute of Physics.

\section{Related Articles}

Controlling activated processes of nonadiabatically, periodically driven dynamical systems: A multiple scale perturbation approach

J. Chem. Phys. 136, 234506 (2012)

Brownian motions on metric graphs

J. Math. Phys. 53, 095206 (2012)

Optimizing hierarchical equations of motion for quantum dissipation and quantifying quantum bath effects on quantum transfer mechanisms

J. Chem. Phys. 136, 224103 (2012)

Force-dependent mobility and entropic rectification in tubes of periodically varying geometry

J. Chem. Phys. 136, 214110 (2012)

Brownian rod scheme in microenvironment sensing

AlP Advances 2, 012180 (2012)

\section{Additional information on J. Math. Phys.}

Journal Homepage: http://jmp.aip.org/

Journal Information: http://jmp.aip.org/about/about_the_journal

Top downloads: http://jmp.aip.org/features/most_downloaded

Information for Authors: http://jmp.aip.org/authors

\section{ADVERTISEMENT}

The most comprehensive support for physics in any mathematical software package World-leading tools for performing calculations in theoretical physics

www.maplesoft.com/physics
Your work in Maple matches how you would write the problems and solutions by hand

- State-of-the-art environment for algebraic computations in physics

The only system with the ability to handle a wide range of physics computations as well as pencil-and-paper style input and textbook-quality display of results

- Access to Maple's full mathematical power, programming language, visualization routines, and document creation tools 


\title{
Translation-covariant Markovian master equation for a test particle in a quantum fluid
}

\author{
Bassano Vacchinia) \\ Dipartimento di Fisica dell'Università di Milano and Istituto Nazionale di Fisica \\ Nucleare, Sezione di Milano, Via Celoria 16, I-20133, Milan, Italy
}

(Received 9 March 2001; accepted for publication 11 May 2001)

\begin{abstract}
A recently proposed master equation in the Lindblad form is studied with respect to covariance properties and existence of a stationary solution. The master equation describes the interaction of a test particle with a quantum fluid, the so-called Rayleigh gas, and is characterized by the appearance of a two-point correlation function known as the dynamic structure factor, which reflects symmetry and statistical mechanics properties of the fluid. In the case of a free gas, all relevant physical parameters such as fugacity, ratio between the masses, momentum transfer, and energy transfer are put into evidence, giving an exact expansion of the dynamic structure factor. The limit in which these quantities are small is then considered. In particular, in the Brownian limit a Fokker-Planck equation is obtained in which the corrections due to quantum statistics can be explicitly evaluated and are given in terms of the Bose function $g_{0}(z)$ and the Fermi function $f_{0}(z)$. (C) 2001 American Institute of Physics. [DOI: 10.1063/1.1386409]
\end{abstract}

\section{INTRODUCTION}

The study of dissipative systems in a quantum mechanical framework is a subject of major interest for many physical communities especially in connection with applications. Recently, however, the subject has gained new interest also for physicists concerned with foundations of quantum mechanics, due to the relevance of the notion of decoherence as a gateway between the classical and quantum worlds. ${ }^{1}$ This interest is strongly supported and partially motivated by a spectacular improvement in many experimental techniques useful for handling with great precision single- or few-particle systems, checking for coherence properties in their dynamical evolution. ${ }^{2}$ In this connection models for quantum dissipation determined by the symmetry properties of the microphysical interaction and by symmetry and statistical mechanics properties of the environment could be of interest for a large class of phenomena. In the Markovian limit quantumdynamical semigroups ${ }^{3}$ seem the most natural quantum mechanical framework for the description of dissipative systems ${ }^{4}$ and a lot of work has been done in this direction, both at rigorous and formal levels, especially with reference to the structural result of Lindblad, which fixes the form of the generator of a completely positive quantum-dynamical semigroup in the case of norm continuity. ${ }^{5}$ In this article we will study in detail some structural properties of a recently proposed Markovian master equation for the description of the dynamics of a test particle in a fluid, ${ }^{6-9}$ the so-called Rayleigh gas. ${ }^{4}$ This simple but realistic model is of particular interest in statistical mechanics, being a paradigmatic example which opens the way to the study of interacting truly many-body systems. In the quantum regime a feature of independent interest is the relevance of quantum statistics of particles making up the fluid, especially in connection with the recent experimental realization of almost noninteracting degenerate gas samples of both Bose and Fermi particles. ${ }^{10}$ The considered master equation was derived by assuming a translationally invariant interaction between the test particle and a homogeneous fluid, and has the general structure of a generator of translation-covariant dynamical semigroups considered in Ref. 11. In this way a direct

${ }^{a)}$ Electronic mail: bassano.vacchini@mi.infn.it 
physical interpretation arises, at least in a particular case, of the general structure given in Ref. 11. Further symmetry or equilibrium properties, which are of fundamental relevance in order to determine the realm of validity of a given master equation, ${ }^{9}$ are shown to be a direct consequence of particular physical features of the environment, embodied in a specific two-point correlation function, the so-called dynamic structure factor. ${ }^{12}$

The article is organized as follows: in Sec. II the master equation is introduced and the property of covariance with respect to translations and rotations is considered with reference to the corresponding symmetries of the environment embodied in its dynamic structure factor; moreover, the existence of a stationary solution is proved, provided the environment is in a $\beta$-KMS state. ${ }^{13}$ In Sec. III the case of a free quantum gas is considered, the dynamic structure factor is explicitly calculated, its exact expansion with respect to the relevant physical parameters is obtained and in the limit of small fugacity $z$ the expression for Maxwell-Boltzmann particles is recovered. In Sec. IV the Brownian limit in which the test particle is much heavier than the particles making up the gas is dealt with, together with the limit of small momentum transfer, leading from the master equation to a Fokker-Planck equation, strongly dependent on the statistics of the gas. In Sec. V the obtained results are briefly summarized and discussed.

\section{GENERAL FEATURES OF THE MASTER EQUATION}

Let us recall the general expression of the master equation proposed in Refs. 7 and 8 for the description of the motion of a test particle in a homogeneous fluid supposed to be at equilibrium, whose properties we are going to study. The obtained result is based on a scattering theory derivation, assuming a translationally invariant interaction in terms of two-particle collisions, and is expected to be valid on a time scale much longer than the relaxation time of the environment. In the Schrödinger picture the master equation is given by

$$
\frac{d \hat{\varrho}}{d t}=\mathcal{M}[\hat{\varrho}]=-\frac{i}{\hbar}\left[\hat{H}_{0}, \hat{\varrho}\right]+\mathcal{L}[\hat{\varrho}],
$$

where $\hat{H}_{0}=\hat{\boldsymbol{p}}^{2} / 2 M$ is the Hamiltonian of the free particle, $M$ being the mass of the test particle, while the dissipative part is given by the following mapping with a Lindblad structure:

$$
\mathcal{L}[\cdot]=\int_{\mathbf{R}^{3}} d \mu(\boldsymbol{q})\left[\hat{U}(\boldsymbol{q}) \sqrt{S(\boldsymbol{q}, \hat{\boldsymbol{p}})} \cdot \sqrt{S(\boldsymbol{q}, \hat{\boldsymbol{p}})} \hat{U}^{\dagger}(\boldsymbol{q})-\frac{1}{2}\{S(\boldsymbol{q}, \hat{\boldsymbol{p}}), \cdot\}\right],
$$

where the integral is over the parameter space of the translation group in momentum space in three dimensions, the parameter $\boldsymbol{q}$ being the momentum transferred in a collision, $\{\hat{A}, \hat{B}\}$ denotes the Jordan product $\hat{A} \circ \hat{B}=\hat{A} \hat{B}+\hat{B} \hat{A}$, and the operator $\hat{\boldsymbol{p}}$ is the generator of translations. The unitary operators $\hat{U}(\boldsymbol{q})=e^{(i / \hbar) \boldsymbol{q} \cdot \hat{x}}, \boldsymbol{q} \in \mathbf{R}^{3}$, are the generators of translations in momentum space or boosts. The positive measure $d \mu(\boldsymbol{q})$ is given by

$$
d \mu(\boldsymbol{q})=\frac{2 \pi}{\hbar}(2 \pi \hbar)^{3} n|\tilde{t}(q)|^{2} d^{3} \boldsymbol{q},
$$

thus being invariant under both rotations and translations. In fact $n$ is the particle density in the macroscopic system, and the function $\widetilde{t}(q)$, given by

$$
\tilde{t}(q)=\int_{\mathbf{R}^{3}} d^{3} x \frac{e^{(i / \hbar) \boldsymbol{q} \cdot \boldsymbol{x}}}{(2 \pi \hbar)^{3}} t(x),
$$

where $q=|\boldsymbol{q}|$ and $x=|\boldsymbol{x}|$, is the Fourier transform with respect to the transferred momentum $\boldsymbol{q}$ of the $\mathrm{T}$ matrix describing the translationally and rotationally invariant interaction between the test particle and the particles of the fluid, which is supposed to be energy independent. The function 
$S(\boldsymbol{q}, \boldsymbol{p})$, which appears operator-valued in (2), is a positive two-point correlation function known as the dynamic structure factor, ${ }^{12}$ given by the Fourier transform with respect to energy transfer $E(\boldsymbol{q}, \boldsymbol{p})$ and momentum transfer $\boldsymbol{q}$ of the time dependent pair correlation function of the fluid

$$
S(\boldsymbol{q}, \boldsymbol{p})=\frac{1}{2 \pi \hbar} \int_{\mathbf{R}} d t \int_{\mathbf{R}^{3}} d^{3} \boldsymbol{x} e^{(i / \hbar)[E(\boldsymbol{q}, \boldsymbol{p}) t-\boldsymbol{q} \cdot \boldsymbol{x}]} G(\boldsymbol{x}, t),
$$

where $G(\boldsymbol{x}, t)$ is the time dependent pair correlation function

$$
G(\boldsymbol{x}, t)=\frac{1}{N} \int_{\mathbf{R}^{3}} d^{3} \boldsymbol{y}\langle N(\boldsymbol{y}) N(\boldsymbol{y}+\boldsymbol{x}, t)\rangle,
$$

$N(\boldsymbol{y})$ being the operator density of particles in the fluid and $\langle\cdots\rangle$ the ensemble average with respect to the state of the macroscopic system. The expression $E(\boldsymbol{q}, \boldsymbol{p})$ gives the energy transfer in a collision where the test particle changes its momentum from $\boldsymbol{p}$ to $\boldsymbol{p}+\boldsymbol{q}$, so that

$$
E(\boldsymbol{q}, \boldsymbol{p})=E_{p+q}-E_{p}=\frac{q^{2}}{2 M}+\frac{\boldsymbol{p} \cdot \boldsymbol{q}}{M}
$$

Note that in (4) we have used as variables momentum and energy transferred to the test particle. In the sequel we will use both the equivalent notations $S(\boldsymbol{q}, \boldsymbol{p})$ and $S(\boldsymbol{q}, E(\boldsymbol{q}, \boldsymbol{p})) \equiv S(\boldsymbol{q}, E)$, according to convenience. The dynamic structure factor is a very important physical quantity, giving the spectrum of spontaneous fluctuations of the system, and it is of direct experimental access: in fact, as first obtained by van Hove in a fundamental work, ${ }^{14}$ it is directly related to the energy dependent differential cross-section per target particle describing scattering of a microscopic probe off a macroscopic sample through the formula

$$
\frac{d^{2} \sigma}{d \Omega_{p^{\prime}} d E_{p^{\prime}}}=(2 \pi \hbar)^{6}\left(\frac{M}{2 \pi \hbar^{2}}\right)^{2} \frac{p^{\prime}}{p}|\tilde{t}(q)|^{2} S(\boldsymbol{q}, E),
$$

referring to scattering of the microscopic probe from $\boldsymbol{p}$ to $\boldsymbol{p}^{\prime}=\boldsymbol{p}+\boldsymbol{q}$. The dynamic structure factor is given in (7) as a function of momentum and energy transfer, which are the measured quantities in scattering experiments, the energy $E$ being related to $\boldsymbol{q}$ and $\boldsymbol{p}$ through (6). The appearance of the dynamic structure factor in (7) gives the physical reason for its being positive definite for every system. The main point in (1) and (2) is the determination of the specific expressions for the measure $d \mu(\boldsymbol{q})$ given by (3), and of the operator valued function $S(\boldsymbol{q}, \cdot)$, given by (4), which can only be obtained on the basis of a microphysical derivation of the equation, relying on some physical model. A general structure encompassing (2) has already been considered by Holevo in a purely mathematical context, studying the general structure of generators of translation-covariant dynamical semigroups. ${ }^{11}$ In particular Holevo has proven that when the generator is bounded it must have a structure of the form (1) with $\mathcal{L}$ given by (2) provided all operators appearing in (1) and (2) are bounded ${ }^{11}$ [see also Refs. 15 and 16, where further restrictions to the structure of (2) appear, to be discussed later on], and allowing, instead of the structure

$$
\hat{U}(\boldsymbol{q}) \sqrt{S(\boldsymbol{q}, \hat{\boldsymbol{p}})} \cdot \sqrt{S(\boldsymbol{q}, \hat{\boldsymbol{p}})} \hat{U}^{\dagger}(\boldsymbol{q})
$$

appearing in (2), where $S(\boldsymbol{q}, \hat{\boldsymbol{p}})$ is self-adjoint and positive, the more general structure

$$
\hat{U}(\boldsymbol{q}) L(\boldsymbol{q}, \hat{\boldsymbol{p}}) \cdot L^{\dagger}(\boldsymbol{q}, \hat{\boldsymbol{p}}) \hat{U}^{\dagger}(\boldsymbol{q}) .
$$

If the generator is unbounded also diffusion terms of the form considered in (37) may appear, and the operators appearing in (1) and (2) may be unbounded (see Ref. 11 for further details). In the 
general model considered here the Hamiltonian is given by the unbounded operator $\hat{H}_{0}$ $=\hat{\boldsymbol{p}}^{2} / 2 \mathrm{M}$, while the remaining part of the generator is determined by the explicit expression of the physical quantities $\tilde{t}(q)$ and $S(\boldsymbol{q}, \boldsymbol{p})$, depending on the specific model for the fluid.

We now consider the behavior of (1) with respect to symmetry transformations. Let us consider a locally compact group $G$ and a unitary representation $\hat{U}(g)$, with $g \in G$, on the Hilbert space of the system. Following Ref. 11 we say that a mapping $\mathcal{M}$ in the Schrödinger picture is $G$-covariant if it commutes with the mapping $\mathcal{U}_{g}[\cdot]=\hat{U}(g) \cdot \hat{U}^{\dagger}(g)$ for all $g \in G$ :

$$
\mathcal{M}\left[\mathcal{U}_{g}[\cdot]\right]=\mathcal{U}_{g}[\mathcal{M}[\cdot]]
$$

Let us first consider the case of spatial translations. Then the unitary operators are given by $\hat{U}(\boldsymbol{a})=e^{-(i / \hbar) \boldsymbol{a} \cdot \hat{\boldsymbol{p}}}$ with $\boldsymbol{a} \in \mathbf{R}^{3}$ and exploiting

$$
\left[\hat{U}(\boldsymbol{a}), \hat{H}_{0}\right]=0,[\hat{U}(\boldsymbol{a}), S(\boldsymbol{q}, \hat{\boldsymbol{p}})]=0,
$$

together with the Weyl CCR,

$$
\hat{U}(\boldsymbol{q}) \hat{U}(\boldsymbol{a})=\hat{U}(\boldsymbol{a}) \hat{U}(\boldsymbol{q}) e^{(i / \hbar) \boldsymbol{a} \cdot \boldsymbol{q}},
$$

one immediately has that the mapping $\mathcal{M}$ given by (1) is translation-covariant. This property goes back to homogeneity of the fluid and translational invariance of the interaction, as can be seen in the derivation of the master equation. ${ }^{7,8}$ We then consider invariance under rotations, so that the relevant set of unitary operators takes the form $\hat{U}(R)$, with $R \in \mathbf{S O}(3)$. In this case exploiting $\left[\hat{U}(R), \hat{H}_{0}\right]=0$ and the relations

$$
\hat{U}^{\dagger}(R) \hat{U}(\boldsymbol{q}) \hat{U}(R)=\hat{U}\left(R^{-1} \boldsymbol{q}\right), \hat{U}^{\dagger}(R) \hat{\boldsymbol{p}} \hat{U}(R)=R \hat{\boldsymbol{p}},
$$

one has that $\mathcal{M}$ is rotation-covariant provided the dynamic structure factor satisfies for $R$ $\in \mathbf{S O}(3)$

$$
S(R \boldsymbol{q}, R \boldsymbol{p})=S(\boldsymbol{q}, \boldsymbol{p}) .
$$

In fact, if (9) holds, one has

$$
\begin{aligned}
\mathcal{M}\left[\mathcal{U}_{R}[\cdot]\right] & =\int_{\mathbf{R}^{3}} d \mu(\boldsymbol{q}) \hat{U}(R)\left[\hat{U}\left(R^{-1} \boldsymbol{q}\right) \sqrt{S(\boldsymbol{q}, R \hat{\boldsymbol{p}})} \cdot \sqrt{S(\boldsymbol{q}, R \hat{\boldsymbol{p}})} \hat{U}^{\dagger}\left(R^{-1} \boldsymbol{q}\right)-\frac{1}{2}\{S(\boldsymbol{q}, R \hat{\boldsymbol{p}}), \cdot\}\right] \hat{U}^{\dagger}(R) \\
& =\int_{\mathbf{R}^{3}} d \mu(\boldsymbol{q}) \hat{U}(R)\left[\hat{U}(\boldsymbol{q}) \sqrt{S(\boldsymbol{q}, \hat{\boldsymbol{p}})} \cdot \sqrt{S(\boldsymbol{q}, \hat{\boldsymbol{p}})} \hat{U}^{\dagger}(\boldsymbol{q})-\frac{1}{2}\{S(\boldsymbol{q}, \hat{\boldsymbol{p}}), \cdot\}\right] \hat{U}^{\dagger}(R) \\
& =\mathcal{U}_{R}[\mathcal{M}[\cdot]] .
\end{aligned}
$$

On the other hand, (9) is directly linked to rotational invariance of the surrounding environment, as one can see observing that $E(\boldsymbol{q}, \boldsymbol{p})$ as given by (6) satisfies

$$
E(R \boldsymbol{q}, R \boldsymbol{p})=E(\boldsymbol{q}, \boldsymbol{p}),
$$

and considering the identity

$$
\begin{aligned}
S(R \boldsymbol{q}, R \boldsymbol{p}) & =\frac{1}{2 \pi \hbar} \int_{\mathbf{R}} d t \int_{\mathbf{R}^{3}} d^{3} \boldsymbol{x} e^{(i / \hbar)[E(R \boldsymbol{q}, R \boldsymbol{p}) t-R \boldsymbol{q} \cdot \boldsymbol{x}]} G(\boldsymbol{x}, t) \\
& =\frac{1}{2 \pi \hbar} \int_{\mathbf{R}} d t \int_{\mathbf{R}^{3}} d^{3} \boldsymbol{x} e^{(i / \hbar)[E(\boldsymbol{q}, \boldsymbol{p}) t-\boldsymbol{q} \cdot \boldsymbol{x}]} G(R \boldsymbol{x}, t)
\end{aligned}
$$


so that (9) holds if and only if the pair correlation function is invariant under rotations,

$$
G(R x, t)=G(x, t) .
$$

In order to proceed further and consider the existence of stationary solutions we make the natural assumption that the state of the macroscopic system, with respect to which the expectation value in (5) is calculated, is a $\beta$-KMS state, so that the relation

$$
\langle\hat{A}(w) \hat{B}\rangle=\langle\hat{B} \hat{A}(w+i \hbar \beta)\rangle
$$

holds. This in turn implies that the dynamic structure factor $S(\boldsymbol{q}, \boldsymbol{p})$ satisfies an identity known as detailed balance condition, ${ }^{12}$

$$
S(\boldsymbol{q}, \boldsymbol{p})=e^{-\beta\left(q^{2} / 2 M+\boldsymbol{p} \cdot \boldsymbol{q} / M\right)} S(-\boldsymbol{q}, \boldsymbol{p}+\boldsymbol{q}),
$$

usually expressed in terms of the dependence on the transferred energy

$$
S(\boldsymbol{q}, E(\boldsymbol{q}, \boldsymbol{p}))=e^{-\beta E(\boldsymbol{q}, \boldsymbol{p})} S(-\boldsymbol{q},-E(\boldsymbol{q}, \boldsymbol{p})),
$$

the sign of the exponential being determined by the fact that we are considering momentum and energy transferred to the particle. It will prove useful for further considerations to introduce a symmetrized version of the dynamic structure factor, given by

$$
\widetilde{S}(\boldsymbol{q}, E)=e^{(\beta / 2) E} S(\boldsymbol{q}, E)
$$

or equivalently

$$
\widetilde{S}(\boldsymbol{q}, \boldsymbol{p})=e^{(\beta / 2)\left(q^{2} / 2 M+\boldsymbol{p} \cdot \boldsymbol{q} / M\right)} S(\boldsymbol{q}, \boldsymbol{p}),
$$

satisfying instead of (12) the more symmetric

$$
\widetilde{S}(\boldsymbol{q}, E)=\widetilde{S}(-\boldsymbol{q},-E),
$$

so that (11) becomes

$$
\widetilde{S}(\boldsymbol{q}, \boldsymbol{p})=\widetilde{S}(-\boldsymbol{q}, \boldsymbol{p}+\boldsymbol{q}) .
$$

We now look for a stationary solution of (1), given that the environment is in a $\beta$-KMS state, so that due to (10) the dynamic structure factor satisfies the detailed balance condition as in (11). According to translation covariance of the generators we look for a solution invariant under translation, of the form $\rho(\hat{\boldsymbol{p}})$. Since $\left[\hat{H}_{0}, \rho(\hat{\boldsymbol{p}})\right]=0, \rho(\hat{\boldsymbol{p}})$ will be a stationary solution of (1) provided

$$
\begin{aligned}
\mathcal{L}[\rho(\hat{\boldsymbol{p}})] & =\int_{\mathbf{R}^{3}} d \mu(\boldsymbol{q})\left[\hat{U}(\boldsymbol{q}) S(\boldsymbol{q}, \hat{\boldsymbol{p}}) \rho(\hat{\boldsymbol{p}}) \hat{U}^{\dagger}(\boldsymbol{q})-S(\boldsymbol{q}, \hat{\boldsymbol{p}}) \rho(\hat{\boldsymbol{p}})\right] \\
& =\int_{\mathbf{R}^{3}} d \mu(\boldsymbol{q})[S(\boldsymbol{q}, \hat{\boldsymbol{p}}-\boldsymbol{q}) \rho(\hat{\boldsymbol{p}}-\boldsymbol{q})-S(\boldsymbol{q}, \hat{\boldsymbol{p}}) \rho(\hat{\boldsymbol{p}})]=0 .
\end{aligned}
$$

Introducing the function

$$
A(\boldsymbol{q}, \boldsymbol{p})=S(\boldsymbol{q}, \boldsymbol{p}-\boldsymbol{q}) \rho(\boldsymbol{p}-\boldsymbol{q})-S(\boldsymbol{q}, \boldsymbol{p}) \rho(\boldsymbol{p}),
$$

the requirement (16) becomes 


$$
\int_{\mathbf{R}^{3}} d \mu(\boldsymbol{q}) A(\boldsymbol{q}, \boldsymbol{p})=0 .
$$

A sufficient condition for (18) to be valid is that $A(\boldsymbol{q}, \boldsymbol{p})$ be an odd function in $\boldsymbol{q}$, and we shall see that this is exactly the case if $\rho(\hat{\boldsymbol{p}})$ has the canonical structure $\rho_{0}(\hat{\boldsymbol{p}})=e^{-\beta \hat{\boldsymbol{p}}^{2} / 2 M}$, with $M$ the mass of the test particle and $\beta$ the inverse temperature of the macroscopic system, as it is to be expected on physical grounds. Let $\rho_{0}(\hat{\boldsymbol{p}})=e^{-\beta \hat{\boldsymbol{p}}^{2} / 2 M}$. Then

$$
\rho_{0}(\boldsymbol{p}-\boldsymbol{q})=\rho_{0}(\boldsymbol{p}) e^{-\beta\left(q^{2} / 2 M-\boldsymbol{p} \cdot \boldsymbol{q} / M\right)},
$$

so that

$$
A(\boldsymbol{q}, \boldsymbol{p})=\rho_{0}(\boldsymbol{p})\left[S(\boldsymbol{q}, \boldsymbol{p}-\boldsymbol{q}) e^{-\beta\left(q^{2} / 2 M-\boldsymbol{p} \cdot \boldsymbol{q} / M\right)}-S(\boldsymbol{q}, \boldsymbol{p})\right]
$$

and exploiting (11)

$$
A(\boldsymbol{q}, \boldsymbol{p})=\rho_{0}(\boldsymbol{p})[S(-\boldsymbol{q}, \boldsymbol{p})-S(\boldsymbol{q}, \boldsymbol{p})],
$$

which is manifestly odd in $\boldsymbol{q}$.

As mentioned earlier the structure given in (2) is a particular realization of the general expression considered in Ref. 11; however, it does not meet the more stringent requirements exhibited by the dissipative mapping considered in Ref. 16. According to Ref. 11 these requirements are unnecessary if one is looking for the most general translation-covariant generator and we shall show that in the present framework they would lead to unphysical results. In fact the structure proposed in Ref. 16 for the dissipative part would take in the Schrödinger picture the form

$$
\mathcal{L}[\cdot]=\int_{\mathbf{R}^{3}} d \mu(\boldsymbol{q})\left[P(\boldsymbol{q}, \hat{\boldsymbol{p}}) \cdot P^{\dagger}(\boldsymbol{q}, \hat{\boldsymbol{p}})-\frac{1}{2}\left\{P^{\dagger}(\boldsymbol{q}, \hat{\boldsymbol{p}}) P(\boldsymbol{q}, \hat{\boldsymbol{p}}), \cdot\right\}\right],
$$

with the further requirement

$$
P^{\dagger}(\boldsymbol{q}, \hat{\boldsymbol{p}})=P(-\boldsymbol{q}, \hat{\boldsymbol{p}}),
$$

which in our case, since

$$
P(\boldsymbol{q}, \hat{\boldsymbol{p}})=\hat{U}(\boldsymbol{q}) \sqrt{S(\boldsymbol{q}, \hat{\boldsymbol{p}})}
$$

is not satisfied, being equivalent to the requirement

$$
\hat{U}(\boldsymbol{q}) \sqrt{S(\boldsymbol{q}, \hat{\boldsymbol{p}})}=\hat{U}(\boldsymbol{q}) \sqrt{S(-\boldsymbol{q}, \hat{\boldsymbol{p}}+\boldsymbol{q})},
$$

which does not hold due to the presence of the factor $e^{-\beta\left(q^{2} / 2 M+p \cdot q / M\right)}$ in (11). If instead of the dynamic structure factor $S(\boldsymbol{q}, \boldsymbol{p})$ one would consider the symmetrized dynamic structure factor $\widetilde{S}(\boldsymbol{q}, \boldsymbol{p})$ given by $(14)$, so that $P(\boldsymbol{q}, \boldsymbol{p})$ in $(21)$ would be replaced by

$$
\widetilde{P}(\boldsymbol{q}, \hat{\boldsymbol{p}})=\hat{U}(\boldsymbol{q}) \sqrt{\widetilde{S}(\boldsymbol{q}, \hat{\boldsymbol{p}}),}
$$

then according to (15) the relation (20) would hold and the dissipative mapping $\mathcal{L}$ would conform to the structure proposed in Ref. 16. In this case, however, one would have the unphysical result that $\rho_{0}(\hat{\boldsymbol{p}})=e^{-\beta \hat{\boldsymbol{p}}^{2} / 2 M}$ is no longer a stationary solution, because

$$
\widetilde{A}(\boldsymbol{q}, \boldsymbol{p})=\widetilde{S}(\boldsymbol{q}, \boldsymbol{p}-\boldsymbol{q}) \rho_{0}(\boldsymbol{p}-\boldsymbol{q})-\widetilde{S}(\boldsymbol{q}, \boldsymbol{p}) \rho_{0}(\boldsymbol{p})
$$


is no longer odd in $\boldsymbol{q}$. Other unphysical features linked to the further restriction (20) will be considered in Sec. IV. Note that these features can only be discovered with reference to a specific structure of a translation-covariant generator determined by starting from some microphysical model. In fact the restriction (20), though unnecessary from a mathematical standpoint, could have proven interesting as well from a physical point of view, thus suggesting the result of Ref. 16 as a useful starting point for phenomenological approaches. This does not seem to be the case, in fact the substitution $S(\boldsymbol{q}, \boldsymbol{p}) \rightarrow \widetilde{S}(\boldsymbol{q}, \boldsymbol{p})$, natural in order to comply with Ref. 16, leads to unphysical results.

\section{EXACT EXPRESSION FOR A FREE QUANTUM GAS}

It is of course of interest to analyze the mapping $\mathcal{M}$ given in (1) for a model in which the dynamic structure factor of the fluid can be explicitly calculated: this is the case for an ideal quantum gas considered at finite temperature $T=1 / \beta k$, where $k$ is Boltzmann's constant, and obeying either Bose or Fermi statistics. Apart from simplicity, the case of a free gas can be of interest also in view of the recent experimental realization of dilute quantum samples of Bose or Fermi particles in the degenerate regime..$^{10}$ The dynamic structure factor for an ideal gas takes the form $^{12}$

$$
S_{\mathrm{B} / \mathrm{F}}(\boldsymbol{q}, \boldsymbol{p})=\frac{1}{n} \int_{\mathbf{R}^{3}} \frac{d^{3} \boldsymbol{k}}{(2 \pi \hbar)^{3}}\left\langle n_{k}\right\rangle_{\mathrm{B} / \mathrm{F}}\left(1 \pm\left\langle n_{k-q}\right\rangle_{\mathrm{B} / \mathrm{F}}\right) \delta\left(\frac{(\boldsymbol{p}+\boldsymbol{q})^{2}}{2 M}+\frac{(\boldsymbol{k}-\boldsymbol{q})^{2}}{2 m}-\frac{\boldsymbol{p}^{2}}{2 M}-\frac{\boldsymbol{k}^{2}}{2 m}\right),
$$

where the indexes B or F and signs + or - refer to Bose or Fermi statistics, respectively, $M$ is the mass of the test particle, $m$ is the mass of the particles making up the gas, $n$ is the density of particles in the gas, and $\left\langle n_{k}\right\rangle_{\mathrm{B} / \mathrm{F}}$ is

$$
\left\langle n_{k}\right\rangle_{\mathrm{B} / \mathrm{F}}=\frac{1}{z^{-1} e^{\beta \epsilon_{k} \mp 1}}, \quad \boldsymbol{\epsilon}_{k}=\frac{\boldsymbol{k}^{2}}{2 m},
$$

where $z$ is the fugacity of the gas, related to the chemical potential $\mu$ by $z=e^{\beta \mu}$. For a Bose gas at finite temperature $0 \leqslant z<1$, while for a Fermi gas $z \geqslant 0$. The integral in (22) can be explicitly calculated both for bosons and fermions, giving the result (A6) obtained in Appendix A:

$$
S_{\mathrm{B} / \mathrm{F}}(\boldsymbol{q}, \boldsymbol{p})=\mp \frac{1}{(2 \pi \hbar)^{3}} \frac{2 \pi m^{2}}{n \beta q} \frac{1}{1-e^{\beta E(\boldsymbol{q}, \boldsymbol{p})}} \log \left[\frac{1 \mp z \exp \left[-(\beta / 8 m)\left(2 m E(\boldsymbol{q}, \boldsymbol{p})+q^{2}\right)^{2} / q^{2}\right]}{1 \mp z \exp \left[-(\beta / 8 m)\left(2 m E(\boldsymbol{q}, \boldsymbol{p})-q^{2}\right)^{2} / q^{2}\right]}\right],
$$

with $q=|\boldsymbol{q}|$. In the same way one can consider the case of a free gas of particles satisfying Maxwell-Boltzmann statistics, thus having

$$
S_{\mathrm{MB}}(\boldsymbol{q}, \boldsymbol{p})=\frac{1}{n} \int_{\mathbf{R}^{3}} \frac{d^{3} \boldsymbol{k}}{(2 \pi \hbar)^{3}}\left\langle n_{k}\right\rangle_{\mathrm{MB}} \delta\left(\frac{(\boldsymbol{p}+\boldsymbol{q})^{2}}{2 M}+\frac{(\boldsymbol{k}-\boldsymbol{q})^{2}}{2 m}-\frac{\boldsymbol{p}^{2}}{2 M}-\frac{\boldsymbol{k}^{2}}{2 m}\right),
$$

with

$$
\left\langle n_{k}\right\rangle_{\mathrm{MB}}=z e^{-\beta \epsilon_{k}},
$$

so that the integral in (24) can also be explicitly calculated giving the expression (A8):

$$
S_{\mathrm{MB}}(\boldsymbol{q}, \boldsymbol{p})=\frac{1}{(2 \pi \hbar)^{3}} \frac{2 \pi m^{2}}{n \beta q} z \exp \left[-\frac{\beta}{8 m} \frac{\left(2 m E(\boldsymbol{q}, \boldsymbol{p})+q^{2}\right)^{2}}{q^{2}}\right] .
$$

A convenient way to write (23) and (25) for later expansions is in terms of the function 


$$
\sigma(\boldsymbol{q}, \boldsymbol{p})=\frac{1}{2 q}\left[q^{2}+2 \alpha M E(\boldsymbol{q}, \boldsymbol{p})\right],
$$

where the ratio $\alpha=m / M$ between the masses of the particles of the gas and of the test particle has been put into evidence, thus obtaining, respectively,

$$
\begin{aligned}
S_{\mathrm{B} / \mathrm{F}}(\boldsymbol{q}, \boldsymbol{p})= & \mp \frac{1}{(2 \pi \hbar)^{3}} \frac{2 \pi m^{2}}{n \beta q} \frac{1}{1-\exp \left[(\beta / 2 m)\left(2 \sigma(\boldsymbol{q}, \boldsymbol{p}) q-q^{2}\right)\right]} \\
& \times \log \left[\frac{1 \mp z \exp \left[-(\beta / 2 m) \sigma^{2}(\boldsymbol{q}, \boldsymbol{p})\right]}{1 \mp z \exp \left[-(\beta / 2 m)(\sigma(\boldsymbol{q}, \boldsymbol{p})-q)^{2}\right]}\right]
\end{aligned}
$$

and

$$
S_{\mathrm{MB}}(\boldsymbol{q}, \boldsymbol{p})=\frac{1}{(2 \pi \hbar)^{3}} \frac{2 \pi m^{2}}{n \beta q} z \exp \left[-\frac{\beta}{2 m} \sigma^{2}(\boldsymbol{q}, \boldsymbol{p})\right] .
$$

We have thus put into evidence all the physical parameters which are of interest in specifying the physical model under consideration and its range of validity: $\boldsymbol{q}, E, \alpha$, and $z$. In this perspective the expression for a gas of Maxwell-Boltzmann particles can also be obtained as expected from the dynamic structure factor for a Bose or Fermi gas in the limit of small fugacity $z$. In fact, starting from (27) and expanding the logarithm up to first order in $z$ one has

$$
\begin{aligned}
S_{\mathrm{B} / \mathrm{F}}(\boldsymbol{q}, \boldsymbol{p}, z \ll 1)= & \frac{1}{(2 \pi \hbar)^{3}} \frac{2 \pi m^{2}}{n \beta q} \frac{z}{1-\exp \left[(\beta / 2 m)\left(2 \sigma(\boldsymbol{q}, \boldsymbol{p}) q-q^{2}\right)\right]} \\
& \times\left\{\exp \left[-\frac{\beta}{2 m} \sigma^{2}(\boldsymbol{q}, \boldsymbol{p})\right]-\exp \left[-\frac{\beta}{2 m}(\sigma(\boldsymbol{q}, \boldsymbol{p})-q)^{2}\right]\right\} \\
= & \frac{1}{(2 \pi \hbar)^{3}} \frac{2 \pi m^{2}}{n \beta q} z \exp \left[-\frac{\beta}{2 m} \sigma^{2}(\boldsymbol{q}, \boldsymbol{p})\right]=S_{\mathrm{MB}}(\boldsymbol{q}, \boldsymbol{p}) .
\end{aligned}
$$

Both (23) and (25) or equivalently (27) and (28) are invariant under rotation, as one can see from the fact that they depend on $\boldsymbol{q}$ and $\boldsymbol{p}$ only through $E(\boldsymbol{q}, \boldsymbol{p})$ and the modulus $q$ of $\boldsymbol{q}$, so that

$$
S_{\mathrm{B} / \mathrm{F}}(R \boldsymbol{q}, R \boldsymbol{p})=S_{\mathrm{B} / \mathrm{F}}(\boldsymbol{q}, \boldsymbol{p}), \quad S_{\mathrm{MB}}(R \boldsymbol{q}, R \boldsymbol{p})=S_{\mathrm{MB}}(\boldsymbol{q}, \boldsymbol{p}),
$$

thus leading to a rotation-covariant mapping $\mathcal{M}$ when substituted in (1). In order to grant the existence of the stationary solution $\rho_{0}(\hat{\boldsymbol{p}})$ we have to check that the obtained expressions satisfy the principle of detailed balance. Starting from (23) we have, setting for simplicity $E(\boldsymbol{q}, \boldsymbol{p})=E$ and inverting the argument of the logarithm,

$$
\begin{aligned}
e^{-\beta E} S_{\mathrm{B} / \mathrm{F}}(-\boldsymbol{q},-E) & =\mp \frac{1}{(2 \pi \hbar)^{3}} \frac{2 \pi m^{2}}{n \beta q} \frac{e^{-\beta E}}{1-e^{-\beta E}} \log \left[\frac{1 \mp z \exp \left[-(\beta / 8 m)\left(-2 m E+q^{2}\right)^{2} / q^{2}\right]}{1 \mp z \exp \left[-(\beta / 8 m)\left(-2 m E-q^{2}\right)^{2} / q^{2}\right]}\right] \\
& =\mp \frac{1}{(2 \pi \hbar)^{3}} \frac{2 \pi m^{2}}{n \beta q} \frac{1}{1-e^{\beta E}} \log \left[\frac{\left.1 \mp z \exp [-\beta / 8 m)\left(2 m E+q^{2}\right)^{2} / q^{2}\right]}{1 \mp z \exp \left[-(\beta / 8 m)\left(2 m E-q^{2}\right)^{2} / q^{2}\right]}\right] \\
& =S_{\mathrm{B} / \mathrm{F}}(\boldsymbol{q}, E),
\end{aligned}
$$

which proves (12). Similarly 


$$
\begin{aligned}
e^{-\beta E} S_{\mathrm{MB}}(-\boldsymbol{q},-E) & =\frac{1}{(2 \pi \hbar)^{3}} \frac{2 \pi m^{2}}{n \beta q} z \exp [-\beta E] \exp \left[-\frac{\beta}{8 m} \frac{\left(-2 m E+q^{2}\right)^{2}}{q^{2}}\right] \\
& =\frac{1}{(2 \pi \hbar)^{3}} \frac{2 \pi m^{2}}{n \beta q} z \exp \left[-\frac{\beta}{8 m} \frac{\left(2 m E+q^{2}\right)^{2}}{q^{2}}\right]=S_{\mathrm{MB}}(\boldsymbol{q}, E) .
\end{aligned}
$$

The master equation (1) for the Rayleigh gas in the case of a free gas of Bose or Fermi particles takes therefore the form

$$
\begin{aligned}
\frac{d \hat{\varrho}}{d t}=\mathcal{M}_{\mathrm{B} / \mathrm{F}}[\hat{\varrho}]= & -\frac{i}{\hbar}\left[\hat{H}_{0}, \hat{\varrho}\right]+\int_{\mathbf{R}^{3}} d \mu(\boldsymbol{q}) \\
& \times\left[\hat{U}(\boldsymbol{q}) \sqrt{S_{\mathrm{B} / \mathrm{F}}(\boldsymbol{q}, \hat{\boldsymbol{p}})} \hat{\varrho} \sqrt{S_{\mathrm{B} / \mathrm{F}}(\boldsymbol{q}, \hat{\boldsymbol{p}})} \hat{U}^{\dagger}(\boldsymbol{q})-\frac{1}{2}\left\{S_{\mathrm{B} / \mathrm{F}}(\boldsymbol{q}, \hat{\boldsymbol{p}}), \hat{\varrho}\right\}\right],
\end{aligned}
$$

with $S_{\mathrm{B} / \mathrm{F}}(\boldsymbol{q}, \boldsymbol{p})$ given explicitly by (23), and a similar expression $\mathcal{M}_{\mathrm{MB}}$ can be considered for a free gas of Maxwell-Boltzmann particles. Both $\mathcal{M}_{\mathrm{B} / \mathrm{F}}$ and $\mathcal{M}_{\mathrm{MB}}$ are translation- and rotationcovariant and admit the same stationary solution with the canonical structure $\rho_{0}(\hat{\boldsymbol{p}})$.

\section{BROWNIAN LIMIT}

In the framework of an ideal gas considered in Sec. III, i.e., referring to $\mathcal{M}_{\mathrm{B} / \mathrm{F}}$ and $\mathcal{M}_{\mathrm{MB}}$, we now want to consider the physically distinguished case in which the test particle is much heavier than the particles making up the gas, so that $\alpha=m / M$ is much smaller than one, the so-called Brownian limit. In order to do this we have to evaluate the dynamic structure factor for a free gas in the case $\alpha \ll 1$. The natural starting points are expressions (27) and (28) in which the factor $\alpha$ has been put into evidence through the function (26). In particular we have the relations

$$
\begin{gathered}
\frac{\beta}{2 m} \sigma^{2}(\boldsymbol{q}, \boldsymbol{p})= \\
\frac{\beta}{8 m} q^{2}+\frac{\beta}{2} \frac{1}{2 M}\left[q^{2}+2 \boldsymbol{p} \cdot \boldsymbol{q}\right]+\frac{\beta}{2} \frac{1}{q^{2}} \alpha \frac{1}{4 M}\left[q^{2}+2 \boldsymbol{p} \cdot \boldsymbol{q}\right]^{2} \\
=\frac{\beta}{8 m} q^{2}+\frac{\beta}{2} E(\boldsymbol{q}, \boldsymbol{p})+\frac{\beta}{2} \frac{m}{q^{2}} E^{2}(\boldsymbol{q}, \boldsymbol{p}), \\
\frac{\beta}{2 m}(\sigma(\boldsymbol{q}, \boldsymbol{p})-q)^{2}=\frac{\beta}{8 m} q^{2}-\frac{\beta}{2} \frac{1}{2 M}\left[q^{2}+2 \boldsymbol{p} \cdot \boldsymbol{q}\right]+\frac{\beta}{2} \frac{1}{q^{2}} \alpha \frac{1}{4 M}\left[q^{2}+2 \boldsymbol{p} \cdot \boldsymbol{q}\right]^{2} \\
=\frac{\beta}{8 m} q^{2}-\frac{\beta}{2} E(\boldsymbol{q}, \boldsymbol{p})+\frac{\beta}{2} \frac{m}{q^{2}} E^{2}(\boldsymbol{q}, \boldsymbol{p}), \\
\frac{\beta}{2 m}\left(2 \sigma(\boldsymbol{q}, \boldsymbol{p}) q-q^{2}\right)=\frac{\beta}{2 M}\left[q^{2}+2 \boldsymbol{p} \cdot \boldsymbol{q}\right]=\beta E(\boldsymbol{q}, \boldsymbol{p}) .
\end{gathered}
$$

The Brownian limit can now be taken neglecting the terms of order $\alpha$ in (30) or equivalently considering small energy transfer, corresponding to a broader time scale, and keeping in (30) only the terms linear in $E$, disregarding higher powers of the energy transfer. The resulting dynamic structure factors, denoted by an index $\infty$, are given by

$$
S_{\mathrm{B} / \mathrm{F}}^{\infty}(\boldsymbol{q}, \boldsymbol{p})=\mp \frac{1}{(2 \pi \hbar)^{3}} \frac{2 \pi m^{2}}{n \beta q} \frac{1}{1-e^{\beta\left[q^{2} / 2 M+\boldsymbol{q} \cdot \boldsymbol{p} / M\right]}} \log \left[\frac{1 \mp z e^{-(\beta / 8 m) q^{2}} e^{-(\beta / 2)\left[q^{2} / 2 M+\boldsymbol{q} \cdot \boldsymbol{p} / M\right]}}{1 \mp z e^{-(\beta / 8 m) q^{2}} e^{+(\beta / 2)\left[q^{2} / 2 M+\boldsymbol{q} \cdot \boldsymbol{p} / M\right]}}\right]
$$


and

$$
S_{\mathrm{MB}}^{\infty}(\boldsymbol{q}, \boldsymbol{p})=\frac{1}{(2 \pi \hbar)^{3}} \frac{2 \pi m^{2}}{n \beta q} z e^{-(\beta / 8 m) q^{2}} e^{-(\beta / 2)\left[q^{2} / 2 M+\boldsymbol{q} \cdot \boldsymbol{p} / M\right]},
$$

respectively. Considering the corresponding expressions in terms of $E(\boldsymbol{q}, \boldsymbol{p})$,

$$
S_{\mathrm{B} / \mathrm{F}}^{\infty}(\boldsymbol{q}, E(\boldsymbol{q}, \boldsymbol{p}))=\mp \frac{1}{(2 \pi \hbar)^{3}} \frac{2 \pi m^{2}}{n \beta q} \frac{1}{1-e^{\beta E(\boldsymbol{q}, \boldsymbol{p})}} \log \left[\frac{1 \mp z e^{-(\beta / 8 m) q^{2}} e^{-(\beta / 2) E(\boldsymbol{q}, \boldsymbol{p})}}{1 \mp z e^{-(\beta / 8 m) q^{2}} e^{+(\beta / 2) E(\boldsymbol{q}, \boldsymbol{p})}}\right]
$$

and

$$
S_{\mathrm{MB}}^{\infty}(\boldsymbol{q}, E(\boldsymbol{q}, \boldsymbol{p}))=\frac{1}{(2 \pi \hbar)^{3}} \frac{2 \pi m^{2}}{n \beta q} z e^{-(\beta / 8 m) q^{2}} e^{-(\beta / 2) E(\boldsymbol{q}, \boldsymbol{p})},
$$

one immediately sees that rotational invariance is preserved in this approximation. One can check that also the detailed balance condition is not spoiled. In fact from (33) one has

$$
\begin{aligned}
e^{-\beta E} S_{\mathrm{B} / \mathrm{F}}^{\infty}(-\boldsymbol{q},-E) & =\mp \frac{1}{(2 \pi \hbar)^{3}} \frac{2 \pi m^{2}}{n \beta q} \frac{e^{-\beta E}}{1-e^{-\beta E}} \log \left[\frac{1 \mp z e^{-(\beta / 8 m) q^{2}} e^{+(\beta / 2) E}}{1 \mp z e^{-(\beta / 8 m) q^{2}} e^{-(\beta / 2) E}}\right] \\
& =\mp \frac{1}{(2 \pi \hbar)^{3}} \frac{2 \pi m^{2}}{n \beta q} \frac{1}{1-e^{\beta E}} \log \left[\frac{1 \mp z e^{-(\beta / 8 m) q^{2}} e^{-(\beta / 2) E}}{1 \mp z e^{-(\beta / 8 m) q^{2}} e^{+(\beta / 2) E}}\right] \\
& =S_{\mathrm{B} / \mathrm{F}}^{\infty}(\boldsymbol{q}, E),
\end{aligned}
$$

and from (34)

$$
e^{-\beta E} S_{\mathrm{MB}}^{\infty}(-\boldsymbol{q},-E)=\frac{1}{(2 \pi \hbar)^{3}} \frac{2 \pi m^{2}}{n \beta q} z e^{-(\beta / 8 m) q^{2}} e^{-\beta E(\boldsymbol{q}, \boldsymbol{p})} e^{(\beta / 2) E(\boldsymbol{q}, \boldsymbol{p})}=S_{\mathrm{MB}}^{\infty}(\boldsymbol{q}, E) .
$$

As a result, in place of (29) we now consider the mapping $\mathcal{M}_{\mathrm{B} / \mathrm{F}}^{\infty}$ :

$$
\begin{aligned}
\frac{d \hat{\varrho}}{d t}=\mathcal{M}_{\mathrm{B} / \mathrm{F}}^{\infty}[\hat{\varrho}]= & -\frac{i}{\hbar}\left[\hat{H}_{0}, \hat{\varrho}\right]+\int_{\mathbf{R}^{3}} d \mu(\boldsymbol{q}) \\
& \times\left[\hat{U}(\boldsymbol{q}) \sqrt{S_{\mathrm{B} / \mathrm{F}}^{\infty}(\boldsymbol{q}, \hat{\boldsymbol{p}})} \hat{\varrho} \sqrt{S_{\mathrm{B} / \mathrm{F}}^{\infty}(\boldsymbol{q}, \hat{\boldsymbol{p}})} \hat{U}^{\dagger}(\boldsymbol{q})-\frac{1}{2}\left\{S_{\mathrm{B} / \mathrm{F}}^{\infty}(\boldsymbol{q}, \hat{\boldsymbol{p}}), \hat{\varrho}\right\}\right],
\end{aligned}
$$

and similarly $\mathcal{M}_{\mathrm{MB}}^{\infty}$ for Maxwell-Boltzmann statistics. $\mathcal{M}_{\mathrm{B} / \mathrm{F}}^{\infty}$ and $\mathcal{M}_{\mathrm{MB}}^{\infty}$ are still translation- and rotation-covariant and admit the same stationary solution with the canonical structure $\rho_{0}(\hat{\boldsymbol{p}})$.

In the master equation (1), or according to the physical system under consideration (29) or (35), the quantum scattering rate or transition probability appears through the dynamic structure factor and the square modulus of the Fourier transform of the T matrix determining the integration measure (3), these quantities being connected to the differential scattering cross-section by (7). In order to pass from the master equation to the related Fokker-Planck equation through a KramersMoyal expansion, as stressed by van Kampen ${ }^{17}$ we need to put into evidence a small parameter governing the size of the fluctuations in the macroscopic system. In our case this parameter is naturally given by the momentum transfer $\boldsymbol{q}$, which through the dynamic structure factor is directly linked to the equilibrium fluctuations of the macroscopic system. Small $\boldsymbol{q}$ means longwavelength fluctuations, corresponding to the macroscopic, long range properties of the environment. It is physically meaningful to consider both approximations $|\boldsymbol{q}| \ll 1$ and $\alpha \ll 1$, or equivalently small energy transfer, together, so that starting from the Maxwell-Boltzmann case, 


$$
\begin{aligned}
\mathcal{M}_{\mathrm{MB}}^{\infty}[\cdot]= & -\frac{i}{\hbar}\left[\hat{H}_{0}, \cdot\right]+z \frac{4 \pi^{2} m^{2}}{\beta \hbar} \int_{\mathbf{R}^{3}} d^{3} \boldsymbol{q} \frac{|\widetilde{t}(q)|^{2}}{q} e^{-(\beta / 8 m)(1+2 \alpha) q^{2}} \\
& \times\left[e^{(i / \hbar) \boldsymbol{q} \cdot \hat{\boldsymbol{x}}} e^{-(\beta / 4 M) \boldsymbol{q} \cdot \hat{\boldsymbol{p}}} \cdot e^{-(\beta / 4 M) \boldsymbol{q} \cdot \hat{\boldsymbol{p}}} e^{-(i / \hbar) \boldsymbol{q} \cdot \hat{\boldsymbol{x}}}-\frac{1}{2}\left\{e^{-(\beta / 2 M) \boldsymbol{q} \cdot \hat{\boldsymbol{p}}} \cdot \cdot\right\}\right]
\end{aligned}
$$

we expand the dissipative part of the mapping in the small parameter $\boldsymbol{q}$. We will expand the operators depending on $\boldsymbol{q}$ up to second order, so as to have contributions at most bilinear in the operators $\hat{\boldsymbol{x}}$ and $\hat{\boldsymbol{p}}$, position and momentum of the Brownian particle. We thus obtain a structure analogous to the classical Fokker-Planck equation, with a friction term linearly proportional to velocity: this class of models is known as quantum Brownian motion. ${ }^{18-20}$ Recalling that $\alpha \ll 1$ and exploiting the symmetry properties of the integration measure the result for the dissipative part is $^{7}$

$$
\begin{aligned}
-z & \frac{2 \pi^{2} m^{2}}{\beta \hbar} \int_{\mathbf{R}^{3}} d^{3} \boldsymbol{q} \frac{|\tilde{t}(q)|^{2}}{q} e^{-(\beta / 8 m) q^{2}} \sum_{i=1}^{3} \boldsymbol{q}_{i}^{2} \\
& \times\left\{\frac{1}{\hbar^{2}}\left[\hat{\boldsymbol{x}}_{i},\left[\hat{\boldsymbol{x}}_{i}, \cdot\right]\right]+\frac{\beta^{2}}{16 M^{2}}\left[\hat{\boldsymbol{p}}_{i},\left[\hat{\boldsymbol{p}}_{i}, \cdot\right]\right]+\frac{i}{\hbar} \frac{\beta}{2 M}\left[\hat{\boldsymbol{x}}_{i},\left\{\hat{\boldsymbol{p}}_{i}, \cdot\right\}\right]\right\},
\end{aligned}
$$

where $i=1,2,3$ denotes Cartesian coordinates. Due to the isotropy of the environment we have $\boldsymbol{q}_{i}^{2}=\frac{1}{3} q^{2}$, so that we can define the coefficients

$$
\begin{gathered}
D_{p p}=\frac{2}{3} \frac{\pi^{2} m^{2}}{\beta \hbar} \int_{\mathbf{R}^{3}} d^{3} \boldsymbol{q}|\widetilde{t}(q)|^{2} q e^{-(\beta / 8 m) q^{2}}, \\
D_{x x}=\left(\frac{\beta \hbar}{4 M}\right)^{2} D_{p p}, \\
\gamma=\left(\frac{\beta}{2 M}\right) D_{p p},
\end{gathered}
$$

and introduce the following mapping describing quantum dissipation:

$$
\mathcal{L}_{\mathrm{QD}}[\cdot]=-\frac{D_{p p}}{\hbar^{2}} \sum_{i=1}^{3}\left[\hat{\boldsymbol{x}}_{i},\left[\hat{\boldsymbol{x}}_{i}, \cdot\right]\right]-\frac{D_{x x}}{\hbar^{2}} \sum_{i=1}^{3}\left[\hat{\boldsymbol{p}}_{i},\left[\hat{\boldsymbol{p}}_{i}, \cdot\right]\right]-\frac{i}{\hbar} \gamma \sum_{i=1}^{3}\left[\hat{\boldsymbol{x}}_{i},\left\{\hat{\boldsymbol{p}}_{i}, \cdot\right\}\right]
$$

thus coming to the Fokker-Planck equation

$$
\frac{d \hat{\varrho}}{d t}=-\frac{i}{\hbar}\left[\hat{H}_{0}, \hat{\varrho}\right]+\mathcal{L}_{\mathrm{MB}}[\hat{\varrho}]=-\frac{i}{\hbar}\left[\hat{H}_{0}, \hat{\varrho}\right]+z \mathcal{L}_{\mathrm{QD}}[\hat{\varrho}]
$$

In view of the result (38) for the Fokker-Planck equation describing the motion of the Brownian particle in a gas obeying Maxwell-Boltzmann statistics, we now look for the corrections to (38) brought about by quantum statistics at finite temperature. As usual we will deal with both Bose and Fermi statistics, exploiting expression (B6) obtained in Appendix B by deriving an exact expansion for $S_{\mathrm{B} / \mathrm{F}}^{\infty}(\boldsymbol{q}, E)$ : 


$$
\begin{aligned}
S_{\mathrm{B} / \mathrm{F}}^{\infty}(\boldsymbol{q}, E)= & S_{\mathrm{MB}}^{\infty}(\boldsymbol{q}, E)\left[\sum_{k=0}^{\infty}( \pm z)^{k}-\frac{\beta}{8 m} q^{2} \sum_{k=1}^{\infty}( \pm)^{k} k z^{k}\right. \\
& \left.+\frac{1}{12}(\beta E)^{2} \sum_{k=1}^{\infty}( \pm)^{k} k z^{k}+\frac{1}{24}(\beta E)^{2} \sum_{k=1}^{\infty}( \pm)^{k} k^{2} z^{k}+O\left(q^{4}\right)\right],
\end{aligned}
$$

where a suitable expansion in the small parameter $\boldsymbol{q}$ has already been performed. Let us first introduce the Bose-Einstein and the Fermi-Dirac functions, ${ }^{21,22}$ given by

$$
g_{\nu}(z)=\frac{1}{\Gamma(\nu)} \int_{0}^{+\infty} d x \frac{x^{\nu-1}}{z^{-1} e^{x}-1}, \quad 0 \leqslant z<1, \nu>0,
$$

and

$$
f_{\nu}(z)=\frac{1}{\Gamma(\nu)} \int_{0}^{+\infty} d x \frac{x^{\nu-1}}{z^{-1} e^{x}+1}, \quad 0 \leqslant z<\infty, \nu>0
$$

respectively, related for integer $\nu$ by $f_{n}(z)=-g_{n}(-z)$. These functions, typically appearing in the quantum statistical mechanics of Bose and Fermi systems, satisfy the recurrence relations

$$
g_{\nu-1}(z)=z \frac{\partial}{\partial z}\left[g_{\nu}(z)\right], \quad f_{\nu-1}(z)=z \frac{\partial}{\partial z}\left[f_{\nu}(z)\right]
$$

so that they can be defined also for $\nu \leqslant 0$. Starting from (42) and exploiting the following representations for $|z|<1$,

$$
g_{\nu}(z)=\sum_{k=1}^{\infty} \frac{z^{k}}{k^{\nu}}, \quad f_{\nu}(z)=\sum_{k=1}^{\infty}(-)^{k-1} \frac{z^{k}}{k^{\nu}},
$$

one can write (39) in the Bose case as

$$
S_{\mathrm{B}}^{\infty}(\boldsymbol{q}, E)=S_{\mathrm{MB}}^{\infty}(\boldsymbol{q}, E)\left[\frac{g_{0}(z)}{z}-\frac{\beta}{8 m} q^{2} g_{-1}(z)+\frac{1}{24}(\beta E)^{2}\left(g_{-2}(z)+2 g_{-1}(z)\right)+O\left(q^{4}\right)\right]
$$

and in the Fermi case as

$$
S_{\mathrm{F}}^{\infty}(\boldsymbol{q}, E)=S_{\mathrm{MB}}^{\infty}(\boldsymbol{q}, E)\left[\frac{f_{0}(z)}{z}+\frac{\beta}{8 m} q^{2} f_{-1}(z)-\frac{1}{24}(\beta E)^{2}\left(f_{-2}(z)+2 f_{-1}(z)\right)+O\left(q^{4}\right)\right],
$$

where the functions appearing in (43) can be written for $0 \leqslant z<1$ in closed form as

$$
\begin{gathered}
g_{0}(z)=\frac{z}{1-z}, \\
g_{-1}(z)=\frac{z}{(1-z)^{2}}, \quad g_{-2}(z)=\frac{z+z^{2}}{(1-z)^{3}},
\end{gathered}
$$

while the functions appearing in (44) can be written for $0 \leqslant z<\infty$ in closed form as

$$
f_{0}(z)=\frac{z}{1+z}
$$




$$
f_{-1}(z)=\frac{z}{(1+z)^{2}}, \quad f_{-2}(z)=\frac{z-z^{2}}{(1+z)^{3}} .
$$

To evaluate the corrections due to quantum statistics we note that when $S_{\mathrm{MB}}^{\infty}(\boldsymbol{q}, E(\boldsymbol{q}, \boldsymbol{p}))$ is substituted by an expression of the form

$$
S_{\mathrm{MB}}^{\infty}(\boldsymbol{q}, E(\boldsymbol{q}, \boldsymbol{p})) A\left[1+2 B q^{2}+2 C(\boldsymbol{p} \cdot \boldsymbol{q})^{2}\right],
$$

so that keeping terms at most quadratic in $\boldsymbol{q}$ in the correction

$$
\sqrt{S_{\mathrm{MB}}^{\infty}(\boldsymbol{q}, E(\boldsymbol{q}, \boldsymbol{p}))} \rightarrow \sqrt{S_{\mathrm{MB}}^{\infty}(\boldsymbol{q}, E(\boldsymbol{q}, \boldsymbol{p}))} \sqrt{A}\left[1+B q^{2}+C(\boldsymbol{p} \cdot \boldsymbol{q})^{2}\right],
$$

the mapping $\mathcal{M}_{\mathrm{MB}}^{\infty}$ always in the same approximation becomes

$$
\begin{aligned}
& -\frac{i}{\hbar}\left[\hat{H}_{0}, \cdot\right]+A \int_{\mathbf{R}^{3}} d \mu(\boldsymbol{q})\left[\hat{U}(\boldsymbol{q}) \sqrt{S_{\mathrm{MB}}^{\infty}(\boldsymbol{q}, \hat{\boldsymbol{p}})} \cdot \sqrt{S_{\mathrm{MB}}^{\infty}(\boldsymbol{q}, \hat{\boldsymbol{p}})} \hat{U}^{\dagger}(\boldsymbol{q})-\frac{1}{2}\left\{S_{\mathrm{MB}}^{\infty}(\boldsymbol{q}, \hat{\boldsymbol{p}}), \cdot\right\}\right] \\
& \quad+2 A B \int_{\mathbf{R}^{3}} d \mu(\boldsymbol{q}) q^{2}\left[\hat{U}(\boldsymbol{q}) \sqrt{S_{\mathrm{MB}}^{\infty}(\boldsymbol{q}, \hat{\boldsymbol{p}})} \cdot \sqrt{S_{\mathrm{MB}}^{\infty}(\boldsymbol{q}, \hat{\boldsymbol{p}})} \hat{U}^{\dagger}(\boldsymbol{q})-\frac{1}{2}\left\{S_{\mathrm{MB}}^{\infty}(\boldsymbol{q}, \hat{\boldsymbol{p}}), \cdot\right\}\right] \\
& \quad+A C \int_{\mathbf{R}^{3}} d \mu(\boldsymbol{q})\left[\hat{U}(\boldsymbol{q}) \sqrt{S_{\mathrm{MB}}^{\infty}(\boldsymbol{q}, \hat{\boldsymbol{p}})}\left\{(\hat{\boldsymbol{p}} \cdot \boldsymbol{q})^{2}, \cdot\right\} \sqrt{S_{\mathrm{MB}}^{\infty}(\boldsymbol{q}, \hat{\boldsymbol{p}})} \hat{U}^{\dagger}(\boldsymbol{q})-\left\{S_{\mathrm{MB}}^{\infty}(\boldsymbol{q}, \hat{\boldsymbol{p}})(\hat{\boldsymbol{p}} \cdot \boldsymbol{q})^{2}, \cdot\right\}\right] .
\end{aligned}
$$

Looking at (47) one immediately sees that, keeping terms at most quadratic in $\boldsymbol{q}$, the last two terms are to be neglected, since the dynamic structure factor $S_{\mathrm{MB}}^{\infty}(\boldsymbol{q}, \hat{\boldsymbol{p}})$ and the unitary operators $\hat{U}(\boldsymbol{q})$ can now only bring in a constant factor. The only change in the structure of the mapping is therefore given by the numerical factor $A$ multiplying the dissipative part. This factor is actually given by $g_{0}(z) / z$ in the Bose case and by $f_{0}(z) / z$ in the Fermi case. The Fokker-Planck equation (38) in the case of Bose statistics of the gas therefore becomes

$$
\frac{d \hat{\varrho}}{d t}=-\frac{i}{\hbar}\left[\hat{H}_{0}, \hat{\varrho}\right]+\mathcal{L}_{\mathrm{B}}[\hat{\varrho}]=-\frac{i}{\hbar}\left[\hat{H}_{0}, \hat{\varrho}\right]+g_{0}(z) \mathcal{L}_{\mathrm{QD}}[\hat{\varrho}]
$$

while for Fermi particles one has

$$
\frac{d \hat{\varrho}}{d t}=-\frac{i}{\hbar}\left[\hat{H}_{0}, \hat{\varrho}\right]+\mathcal{L}_{\mathrm{F}}[\hat{\varrho}]=-\frac{i}{\hbar}\left[\hat{H}_{0}, \hat{\varrho}\right]+f_{0}(z) \mathcal{L}_{\mathrm{QD}}[\hat{\varrho}]
$$

and the following simple relations hold:

$$
\begin{gathered}
\mathcal{L}_{\mathrm{MB}}=z \mathcal{L}_{\mathrm{QD}}, \\
\mathcal{L}_{\mathrm{B}}=g_{0}(z) \mathcal{L}_{\mathrm{QD}}=\frac{z}{1-z} \mathcal{L}_{\mathrm{QD}}=\frac{1}{1-z} \mathcal{L}_{\mathrm{MB}}, \\
\mathcal{L}_{\mathrm{F}}=f_{0}(z) \mathcal{L}_{\mathrm{QD}}=\frac{z}{1+z} \mathcal{L}_{\mathrm{QD}}=\frac{1}{1+z} \mathcal{L}_{\mathrm{MB}} .
\end{gathered}
$$

According to (50) and setting after (36)

$$
\gamma_{\mathrm{MB}}=z \gamma=z \frac{\beta}{2 M} D_{p p}=z \frac{1}{3} \frac{\pi^{2} m^{2}}{M \hbar} \int d^{3} \boldsymbol{q}|\tilde{t}(q)|^{2} q e^{-(\beta / 8 m) q^{2}},
$$


one has the following very simple relation between the friction coefficients in (38) and (48) or (49):

$$
\gamma_{\mathrm{B} / \mathrm{F}}=\frac{\gamma_{\mathrm{MB}}}{1 \mp z}
$$

The relationship between the Fokker-Planck equations for Maxwell-Boltzmann or Bose and Fermi statistics, as given, respectively, by (38), (48) and (49), is actually remarkably simple: they have the very same operator structure, apart from an overall coefficient depending on the fugacity of the gas, which determines the relative weight of the dissipative contribution to the dynamics. As it is to be expected, only the statistics of the reservoir is of relevance, since the test particle is a single particle. The Fokker-Planck equations obtained for the description of quantum dissipation may be compactly written:

$$
\frac{d \hat{\varrho}}{d t}=-\frac{i}{\hbar}\left[\hat{H}_{0}, \hat{\varrho}\right]+\zeta(z) \mathcal{L}_{\mathrm{QD}}[\hat{\varrho}]
$$

with $\zeta(z)$ defined as follows:

$$
\zeta(z)=\left\{\begin{array}{lc}
z, & \text { Maxwell-Boltzmann, } \\
z /(1-z), & \text { Bose, } \\
z /(1+z) & \text { Fermi. }
\end{array}\right.
$$

We now briefly come back to the question dealt with at the end of Sec. II about the physical relevance of the structure of the translation-covariant master equation obtained in Ref. 16. As already stressed, the master equation (1), while having the general translation-covariant structure considered in Ref. 11, does not comply with the further restrictions given in Ref. 16, while this would be the case if instead of the dynamic structure factor $S(\boldsymbol{q}, \boldsymbol{p})$ one would consider the symmetrized correlation function $\widetilde{S}(\boldsymbol{q}, \boldsymbol{p})$, which is an even function of $\boldsymbol{q}$ and $E(\boldsymbol{q}, \boldsymbol{p})$. This could be considered a natural phenomenological ansatz in view of the result obtained in Ref. 16. In Sec. II we showed, however, that this substitution would spoil the existence of the expected stationary solution. More than this, if we now consider the Brownian limit, the symmetrized version of $S_{\mathrm{MB}}^{\infty}(\boldsymbol{q}, \hat{\boldsymbol{p}})$, which can be immediately obtained from (34), reads

$$
\widetilde{S}_{\mathrm{MB}}^{\infty}(\boldsymbol{q}, E(\boldsymbol{q}, \boldsymbol{p}))=\frac{1}{(2 \pi \hbar)^{3}} \frac{2 \pi m^{2}}{n \beta q} z e^{-(\beta / 8 m) q^{2}},
$$

so that the dependence on $\boldsymbol{p}$ is completely lost and the whole operator structure in (37) and (38) is washed out, apart from the double commutator with the position operators $\hat{\boldsymbol{x}}_{i}$. In particular the friction term is missing, so that, even though a Lindblad structure is retained, only a completely different physics can be described. In the same way, for the Bose or Fermi dynamic structure factor in the Brownian limit one has from (B6)

$$
\begin{aligned}
\widetilde{S}_{\mathrm{B} / \mathrm{F}}^{\infty}(\boldsymbol{q}, E)= & \widetilde{S}_{\mathrm{MB}}^{\infty}(\boldsymbol{q}, E)\left[\sum_{k=0}^{\infty}( \pm z)^{k}-\frac{\beta}{8 m} q^{2} \sum_{k=1}^{\infty}( \pm)^{k} k z^{k}\right. \\
& \left.+\frac{1}{12}(\beta E)^{2} \sum_{k=1}^{\infty}( \pm)^{k} k z^{k}+\frac{1}{24}(\beta E)^{2} \sum_{k=1}^{\infty}( \pm)^{k} k^{2} z^{k}+O\left(q^{4}\right)\right],
\end{aligned}
$$


and, once again, recalling (47) written in terms of $\widetilde{S}_{\mathrm{MB}}^{\infty}$ rather than $S_{\mathrm{MB}}^{\infty}$, one sees that under the same approximations as before the operator structure in the dissipative part of both (48) and (49) is washed out apart from the contribution due to the double commutator in the position operators of the particle $\hat{\boldsymbol{x}}_{i}$.

We now consider some structural features of the mapping $\mathcal{L}_{\mathrm{QD}}$ given by (37) in terms of which the Fokker-Planck equation (53) encompassing all three statistics is given. $G$-covariance of $\mathcal{L}_{\mathrm{QD}}$ under translations and rotations immediately follows from its very structure and the transformation laws for the operators $\hat{\boldsymbol{x}}$ and $\hat{\boldsymbol{p}}$ :

$$
\hat{U}^{\dagger}(\boldsymbol{a}) \hat{\boldsymbol{x}} \hat{U}(\boldsymbol{a})=\hat{\boldsymbol{x}}+\boldsymbol{a}, \quad \hat{U}^{\dagger}(\boldsymbol{a}) \hat{\boldsymbol{p}} \hat{U}(\boldsymbol{a})=\hat{\boldsymbol{p}}, \quad \hat{U}^{\dagger}(R) \hat{\boldsymbol{x}} \hat{U}(R)=R \hat{\boldsymbol{x}}, \quad \hat{U}^{\dagger}(R) \hat{\boldsymbol{p}} \hat{U}(R)=R \hat{\boldsymbol{p}} .
$$

One can also see that an operator with the expected canonical structure is a stationary solution of (53) in that

$$
\mathcal{L}_{\mathrm{QD}}\left[\rho_{0}(\hat{\boldsymbol{p}})\right]=0,
$$

due to the relationship

$$
\frac{\gamma}{D_{p p}}=\frac{\beta}{2 M}
$$

obeyed by the coefficients defined in (36) and entering in (37). A few more remarks are in order. The typical structure of translation-covariant mappings describing quantum dissipation in analogy with the classical Fokker-Planck equation that one finds in the physical literature is given by ${ }^{23}$

$$
\mathcal{L}_{\mathrm{FP}}^{\chi}[\cdot]=-\frac{i}{\hbar} \gamma \sum_{i=1}^{3}\left[\hat{\boldsymbol{x}}_{i},\left\{\hat{\boldsymbol{p}}_{i}, \cdot\right\}\right]-\frac{1}{\hbar^{2}} \frac{2 M \gamma}{\beta} \sum_{i=1}^{3}\left[\hat{\boldsymbol{x}}_{i},\left[\hat{\boldsymbol{x}}_{i}, \cdot\right]\right]-\chi \frac{\beta \gamma}{M} \sum_{i=1}^{3}\left[\hat{\boldsymbol{p}}_{i},\left[\hat{\boldsymbol{p}}_{i}, \cdot\right]\right]
$$

where the ratio between the first two coefficients, given by $\beta / 2 M$ as in (55), is fixed by the requirement that $\rho_{0}(\hat{\boldsymbol{p}})$ be a stationary solution, i.e., $\mathcal{L}_{\mathrm{FP}}^{\chi}\left[\rho_{0}(\hat{\boldsymbol{p}})\right]=0$, and the only freedom left, apart from the overall multiplying coefficient $\gamma$, is given by the adimensional factor $\chi$. If one further asks that (56) can be cast in Lindblad form, so that $\mathcal{L}{ }_{\mathrm{FP}}^{\chi}$ is the generator of a completely positive dynamical semigroup, ${ }^{19}$ one has the further simple requirement ${ }^{18,24}$

$$
\chi \geqslant \frac{1}{8}
$$

In fact under this condition, observing that for the operators

$$
\hat{\boldsymbol{B}}_{i \pm}=\hat{\boldsymbol{x}}_{i} \pm i \kappa \hat{\boldsymbol{p}}_{i}
$$

we have the identity

$$
\hat{\boldsymbol{B}}_{i \pm} \cdot \hat{\boldsymbol{B}}_{i \pm}^{\dagger}-\frac{1}{2}\left\{\hat{\boldsymbol{B}}_{i \pm}^{\dagger} \hat{\boldsymbol{B}}_{i \pm}, \cdot\right\}=-\frac{1}{2}\left\{\left[\hat{\boldsymbol{x}}_{i},\left[\hat{\boldsymbol{x}}_{i}, \cdot\right]\right]+\kappa^{2}\left[\hat{\boldsymbol{p}}_{i},\left[\hat{\boldsymbol{p}}_{i}, \cdot\right]\right] \pm 2 i \kappa\left[\hat{\boldsymbol{x}}_{i},\left\{\hat{\boldsymbol{p}}_{i}, \cdot\right\}\right] \mp i \kappa\left[\left\{\hat{\boldsymbol{x}}_{i}, \hat{\boldsymbol{p}}_{i}\right\}, \cdot\right]\right\},
$$

we may write $\mathcal{L}_{\mathrm{FP}}^{\chi}$ in an explicit Lindblad form in terms of the two generators

$$
\hat{\boldsymbol{L}}_{i+}=\hat{\boldsymbol{x}}_{i}+i \frac{\hbar \beta}{M} \sqrt{\frac{\chi}{2}} \hat{\boldsymbol{p}}_{i}, \quad \hat{\boldsymbol{L}}_{i-}=\hat{\boldsymbol{x}}_{i}-i \frac{\hbar \beta}{M} \sqrt{\frac{\chi}{2}} \hat{\boldsymbol{p}}_{i}
$$

according to 


$$
\begin{gathered}
\mathcal{L}_{\mathrm{FP}}^{\chi}[\cdot]=+\frac{2 \gamma M}{\hbar^{2} \beta}\left(1+\sqrt{\frac{1}{8 \chi}}\right) \sum_{i=1}^{3}\left[\hat{\boldsymbol{L}}_{i+} \cdot \hat{\boldsymbol{L}}_{i+}^{\dagger}-\frac{1}{2}\left\{\hat{\boldsymbol{L}}_{i+}^{\dagger} \hat{\boldsymbol{L}}_{i+}, \cdot\right\}\right] \\
+\frac{2 \gamma M}{\hbar^{2} \beta}\left(1-\sqrt{\frac{1}{8 \chi}}\right) \sum_{i=1}^{3}\left[\hat{\boldsymbol{L}}_{i-} \cdot \hat{\boldsymbol{L}}_{i-}^{\dagger}-\frac{1}{2}\left\{\hat{\boldsymbol{L}}_{i-}^{\dagger} \hat{\boldsymbol{L}}_{i-}, \cdot\right\}\right]-\frac{i}{\hbar} \frac{\gamma}{2} \sum_{i=1}^{3}\left[\left\{\hat{\boldsymbol{x}}_{i}, \hat{\boldsymbol{p}}_{i}\right\}, \cdot\right] .
\end{gathered}
$$

The Fokker-Planck structure $\mathcal{L}_{\mathrm{QD}}$ falls within this class, with the coefficient $\gamma$ given by (36) in terms of a suitable integral of the Fourier transform of the $\mathrm{T}$ matrix describing collisions at microphysical level. Moreover, it corresponds to the value $\chi=\frac{1}{8}$ in (57), so that

$$
\mathcal{L}_{\mathrm{QD}}=\mathcal{L}_{\mathrm{FP}}^{1 / 8}
$$

This in turn implies that $\mathcal{L}_{\mathrm{QD}}$ can be written in a manifest Lindblad form in terms of a single generator for each Cartesian direction. We make the choice ${ }^{7,24}$

$$
\hat{\boldsymbol{a}}_{i}=\frac{\sqrt{2}}{\lambda_{M}}\left(\hat{\boldsymbol{x}}_{i}+\frac{i}{\hbar} \frac{\lambda_{M}^{2}}{4} \hat{\boldsymbol{p}}_{i}\right),
$$

where $\lambda_{M}=\sqrt{\hbar^{2} \beta / M}$, the thermal wavelength associated to the Brownian particle, is put into evidence, so that one has the commutation relations

$$
\left[\hat{\boldsymbol{a}}_{i}, \hat{\boldsymbol{a}}_{j}^{\dagger}\right]=\delta_{i j} .
$$

In such a way we have the alternative expression

$$
\mathcal{L}_{\mathrm{QD}}[\cdot]=-\frac{D_{p p}}{\hbar^{2}} \frac{\lambda_{M}^{2}}{4} \sum_{i=1}^{3} \frac{i}{\hbar}\left[\left\{\hat{\boldsymbol{x}}_{i}, \hat{\boldsymbol{p}}_{i}\right\}, \cdot\right]+\frac{D_{p p}}{\hbar^{2}} \lambda_{M}^{2} \sum_{i=1}^{3}\left[\hat{\boldsymbol{a}}_{i} \cdot \hat{\boldsymbol{a}}_{i}^{\dagger}-\frac{1}{2}\left\{\hat{\boldsymbol{a}}_{i}^{\dagger} \hat{\boldsymbol{a}}_{i}, \cdot\right\}\right],
$$

in which the single generator feature is put into evidence.

\section{CONCLUSIONS AND REMARKS}

In this article we have considered the behavior with respect to covariance under translations and rotations, and the existence of a stationary solution of a recently proposed master equation (1) for the description of the interaction of a test particle with a fluid, a physical model corresponding to the so-called Rayleigh gas. The key result in (1) is the appearance of a two-point correlation function known as dynamic structure factor and given by (4), the general structure conforming to results already obtained in the mathematical literature for the generator of a translation-covariant dynamical semigroup. This correlation function, depending on symmetry and statistical mechanics properties of the fluid, directly determines the behavior of the master equation with respect to covariance under translations and rotations, and existence of a stationary solution with the expected canonical form. Considering the specific case of a free gas, the dynamic structure factor has been explicitly evaluated for Bose, Fermi, and Maxwell-Boltzmann statistics, and the dependence on the physical parameters determining the peculiar features of the model under consideration has been put into evidence in an exact expansion of the dynamic structure function. These parameters are the fugacity of the gas $z$, the ratio between mass of the gas particles and of the test particle $\alpha$, the transferred momentum $\boldsymbol{q}$, and the transferred energy $E(\boldsymbol{q}, \boldsymbol{p})$. Stability of the covariance properties of the master equation and of the existence of a stationary solution is then considered in the limit in which these parameters are small, together with the different explicit expressions of the master equation. In particular, in the Brownian limit $\alpha \ll 1$ and considering small momentum transfer, corresponding through the physical interpretation of the dynamic structure factor to long-wavelength fluctuations, a Fokker-Planck equation with a Lindblad structure is obtained, given by (53), where the results corresponding to Bose, Fermi, and Maxwell-Boltzmann statistics 
are jointly considered. The correction due to quantum statistics in the Fokker-Planck equation is simply expressed through the Bose and Fermi functions given by (45) and (46), respectively.

\section{ACKNOWLEDGMENTS}

The author would like to thank Professor L. Lanz for his support during the whole work and Professor A. Barchielli and Professor A. S. Holevo for useful suggestions. He also thanks Dr. F. Belgiorno for careful reading of the manuscript. This work was supported by MURST under Cofinanziamento and Progetto Giovani.

\section{APPENDIX A: DERIVATION OF EQ. (23) AND EQ. (25)}

In this Appendix we want to explicitly calculate the expression of the dynamic structure factor for a free gas as a function of $\boldsymbol{q}$ and $\boldsymbol{p}$. Working at finite temperature we can carry out the calculation for both Bose and Fermi particles at the same time adopting the convention that the symbol \pm means a + sign for Bose particles and - for Fermi particles. We start from the expression

$$
S_{\mathrm{B} / \mathrm{F}}(\boldsymbol{q}, \boldsymbol{p})=\frac{1}{n} \int_{\mathbf{R}^{3}} \frac{d^{3} \boldsymbol{k}}{(2 \pi \hbar)^{3}}\left\langle n_{k}\right\rangle_{\mathrm{B} / \mathrm{F}}\left(1 \pm\left\langle n_{k-q}\right\rangle_{\mathrm{B} / \mathrm{F}}\right) \delta\left(\frac{(\boldsymbol{p}+\boldsymbol{q})^{2}}{2 M}+\frac{(\boldsymbol{k}-\boldsymbol{q})^{2}}{2 m}-\frac{\boldsymbol{p}^{2}}{2 M}-\frac{\boldsymbol{k}^{2}}{2 m}\right)
$$

with

$$
\left\langle n_{k}\right\rangle_{\mathrm{B} / \mathrm{F}}=\frac{1}{z^{-1} e^{\beta \epsilon_{k} \mp 1}}, \quad \boldsymbol{\epsilon}_{k}=\frac{\boldsymbol{k}^{2}}{2 m}
$$

which can be found, for example, in Ref. 12 and corresponds to (4) for a free gas apart from a singular term proportional, in the continuum limit considered here, to $\delta^{3}(\boldsymbol{q})$, relevant only for $\boldsymbol{q}$ $=0$ and not contributing to the master equation. In fact, in the derivation of the master equation the contributions for $\boldsymbol{q}=0$ exactly cancel out. This term according to (7) corresponds to forward scattering. We now have to evaluate the integral in $\boldsymbol{k}$. This is most easily done writing $S_{\mathrm{B} / \mathrm{F}}(\boldsymbol{q}, \boldsymbol{p})$ in the form

$$
S_{\mathrm{B} / \mathrm{F}}(\boldsymbol{q}, \boldsymbol{p})=\frac{1}{n} \int_{\mathbf{R}^{3}} \frac{d^{3} \boldsymbol{k}}{(2 \pi \hbar)^{3}}\left\langle n_{k}\right\rangle_{\mathrm{B} / \mathrm{F}}\left(1 \pm\left\langle n_{k-q}\right\rangle_{\mathrm{B} / \mathrm{F}}\right) \delta\left(E(\boldsymbol{q}, \boldsymbol{p})+\boldsymbol{\epsilon}_{k-q}-\boldsymbol{\epsilon}_{k}\right)
$$

and observing that

$$
\left\langle n_{k}\right\rangle_{\mathrm{B} / \mathrm{F}}\left(1 \pm\left\langle n_{k-q}\right\rangle_{\mathrm{B} / \mathrm{F}}\right)=\frac{1}{1-e^{\beta\left(\epsilon_{k}-\epsilon_{k-q}\right)}}\left(\left\langle n_{k}\right\rangle_{\mathrm{B} / \mathrm{F}}-\left\langle n_{k-q}\right\rangle_{\mathrm{B} / \mathrm{F}}\right)
$$

so that one has

$$
\begin{aligned}
& S_{\mathrm{B} / \mathrm{F}}(\boldsymbol{q}, \boldsymbol{p})=\frac{1}{n} \frac{1}{1-e^{\beta E(\boldsymbol{q}, \boldsymbol{p})}} \int_{\mathbf{R}^{3}} \frac{d^{3} \boldsymbol{k}}{(2 \pi \hbar)^{3}}\left(\left\langle n_{k}\right\rangle_{\mathrm{B} / \mathrm{F}}-\left\langle n_{k-q}\right\rangle_{\mathrm{B} / \mathrm{F}}\right) \delta\left(E(\boldsymbol{q}, \boldsymbol{p})+\boldsymbol{\epsilon}_{k-q}-\boldsymbol{\epsilon}_{k}\right) \\
& =\frac{1}{n} \frac{1}{1-e^{\beta E(\boldsymbol{q}, \boldsymbol{p})}} \int_{\mathbf{R}^{3}} \frac{d^{3} \boldsymbol{k}}{(2 \pi \hbar)^{3}}\left\{\left\langle n_{k}\right\rangle_{\mathrm{B} / \mathrm{F}} \delta\left(E(\boldsymbol{q}, \boldsymbol{p})+\boldsymbol{\epsilon}_{k-q}-\boldsymbol{\epsilon}_{k}\right)\right. \\
& \left.-\left\langle n_{k}\right\rangle_{\mathrm{B} / \mathrm{F}} \delta\left(E(\boldsymbol{q}, \boldsymbol{p})+\boldsymbol{\epsilon}_{k}-\boldsymbol{\epsilon}_{k+q}\right)\right\} \\
& =\frac{1}{n} \frac{2 m}{1-e^{\beta E(\boldsymbol{q}, \boldsymbol{p})}} \int_{\mathbf{R}^{3}} \frac{d^{3} \boldsymbol{k}}{(2 \pi \hbar)^{3}}\left\langle n_{k}\right\rangle_{\mathrm{B} / \mathrm{F}}\left\{\delta\left(2 m E(\boldsymbol{q}, \boldsymbol{p})+q^{2}-2 \boldsymbol{k} \cdot \boldsymbol{q}\right)\right. \\
& \left.-\delta\left(2 m E(\boldsymbol{q}, \boldsymbol{p})-q^{2}-2 \boldsymbol{k} \cdot \boldsymbol{q}\right)\right\} \text {. }
\end{aligned}
$$


We are thus led to consider an integral of the form

$$
\int_{\mathbf{R}^{3}} d^{3} \boldsymbol{k}\left\langle n_{k}\right\rangle_{\mathrm{B} / \mathrm{F}} \delta(\eta-2 \boldsymbol{k} \cdot \boldsymbol{q})
$$

with $\eta$ a real parameter. Denoting by $\xi$ the cosine of the angle between $\boldsymbol{k}$ and $\boldsymbol{q}$ the integral in (A2) becomes

$$
\begin{aligned}
2 \pi \int_{-1}^{1} d \xi \int_{0}^{+\infty} d k k^{2}\left\langle n_{k}\right\rangle_{\mathrm{B} / \mathrm{F}} \delta(\eta-2 \xi k q) \\
\quad=\int_{-1}^{1} d \xi \int_{0}^{+\infty} d k k^{2}\left\langle n_{k}\right\rangle_{\mathrm{B} / \mathrm{F}} \int_{-\infty}^{+\infty} d p e^{i p(\eta-2 \xi k q)} \\
\quad=\int_{-\infty}^{+\infty} d k k\left\langle n_{k}\right\rangle_{\mathrm{B} / \mathrm{F}} \int_{-\infty}^{+\infty} d p \frac{e^{i p(\eta+2 k q)}}{i 2 p q} .
\end{aligned}
$$

and, using the identity

$$
k\left\langle n_{k}\right\rangle_{\mathrm{B} / \mathrm{F}}= \pm \frac{m}{\beta} \frac{d}{d k} \log \left[1 \mp z e^{-\epsilon_{k}}\right],
$$

we get, integrating by parts,

$$
\begin{aligned}
\int_{\mathbf{R}^{3}} d^{3} \boldsymbol{k}\left\langle n_{k}\right\rangle_{\mathrm{B} / \mathrm{F}} \delta(\eta-2 \boldsymbol{k} \cdot \boldsymbol{q}) & =\mp \frac{2 \pi m}{\beta} \int_{-\infty}^{+\infty} d k \log \left[1 \mp z e^{-\epsilon_{k}}\right] \int_{-\infty}^{+\infty} \frac{d p}{2 \pi} e^{i p(\eta+2 k q)} \\
& =\mp \frac{\pi m}{\beta q} \log \left\{1 \mp z \exp \left[-\frac{\beta}{8 m}\left(\frac{\eta}{q}\right)^{2}\right]\right\}
\end{aligned}
$$

Inserting the result (A5) in (A1) one immediately has

$$
S_{\mathrm{B} / \mathrm{F}}(\boldsymbol{q}, \boldsymbol{p})=\mp \frac{1}{(2 \pi \hbar)^{3}} \frac{2 \pi m^{2}}{n \beta q} \frac{1}{1-e^{\beta E(\boldsymbol{q}, \boldsymbol{p})}} \log \left[\frac{\left.1 \mp z \exp \left[-(\beta / 8 m)\left(2 m E(\boldsymbol{q}, \boldsymbol{p})+q^{2}\right)^{2} / q^{2}\right)\right]}{1 \mp z \exp \left[-(\beta / 8 m)\left(2 m E(\boldsymbol{q}, \boldsymbol{p})-q^{2}\right)^{2} / q^{2}\right]}\right] .
$$

In a similar way, starting from the expression of a gas of Maxwell-Boltzmann particles

$$
S_{\mathrm{MB}}(\boldsymbol{q}, \boldsymbol{p})=\frac{1}{n} \int_{\mathbf{R}^{3}} \frac{d^{3} \boldsymbol{k}}{(2 \pi \hbar)^{3}}\left\langle n_{k}\right\rangle_{\mathrm{MB}} \delta\left(\frac{(\boldsymbol{p}+\boldsymbol{q})^{2}}{2 M}+\frac{(\boldsymbol{k}-\boldsymbol{q})^{2}}{2 m}-\frac{\boldsymbol{p}^{2}}{2 M}-\frac{\boldsymbol{k}^{2}}{2 m}\right),
$$

with

$$
\left\langle n_{k}\right\rangle_{\mathrm{MB}}=z e^{-\beta \epsilon_{k}},
$$

we write it in the form

$$
\begin{aligned}
S_{\mathrm{MB}}(\boldsymbol{q}, \boldsymbol{p}) & =\frac{1}{n} \int_{\mathbf{R}^{3}(2 \pi \hbar)^{3}}\left\langle n_{k}\right\rangle_{\mathrm{MB}} \delta\left(E(\boldsymbol{q}, \boldsymbol{p})+\boldsymbol{\epsilon}_{k-q}-\boldsymbol{\epsilon}_{k}\right) \\
& =\frac{2 m}{n} \int_{\mathbf{R}^{3}} \frac{d^{3} \boldsymbol{k}}{(2 \pi \hbar)^{3}}\left\langle n_{k}\right\rangle_{\mathrm{MB}} \delta\left(2 m E(\boldsymbol{q}, \boldsymbol{p})+q^{2}-2 \boldsymbol{k} \cdot \boldsymbol{q}\right) .
\end{aligned}
$$

Analogously to (A2) we have to consider 


$$
\int_{\mathbf{R}^{3}} d^{3} \boldsymbol{k}\left\langle n_{k}\right\rangle_{\mathrm{MB}} \delta(\eta-2 \boldsymbol{k} \cdot \boldsymbol{q})
$$

which according to (A3) becomes

$$
\int_{-\infty}^{+\infty} d k k\left\langle n_{k}\right\rangle_{\mathrm{MB}} \int_{-\infty}^{+\infty} d p \frac{e^{i p(\eta+2 k q)}}{i 2 p q} .
$$

Exploiting instead of (A4) the relation

$$
k\left\langle n_{k}\right\rangle_{\mathrm{MB}}=-\frac{m}{\beta} \frac{d}{d k} z e^{-\epsilon_{k}}
$$

we obtain

$$
\int_{\mathbf{R}^{3}} d^{3} \boldsymbol{k}\left\langle n_{k}\right\rangle_{\mathrm{MB}} \delta(\eta-2 \boldsymbol{k} \cdot \boldsymbol{q})=\frac{\pi m}{\beta q} z \exp \left[-\frac{\beta}{8 m}\left(\frac{\eta}{q}\right)^{2}\right],
$$

which has to be substituted in (A7) leading to

$$
S_{\mathrm{MB}}(\boldsymbol{q}, \boldsymbol{p})=\frac{1}{(2 \pi \hbar)^{3}} \frac{2 \pi m^{2}}{n \beta q} z \exp \left[-\frac{\beta}{8 m} \frac{\left(2 m E(\boldsymbol{q}, \boldsymbol{p})+q^{2}\right)^{2}}{q^{2}}\right] .
$$

\section{APPENDIX B: EXACT EXPANSION OF $S_{\mathrm{B} / \mathrm{F}}$ AND DERIVATION OF EQ. (39)}

We will now derive an expression for $S_{\mathrm{B} / \mathrm{F}}(\boldsymbol{q}, \boldsymbol{p})$ equivalent to (27), in which, however, a series expansion in powers of the fugacity $z$ is put into evidence. The starting point is the Taylor expansion for the logarithm $\log (1+x)=\sum_{k=1}^{\infty}(-)^{k+1} x^{k} / k$, which leads us to write (27) in the form

$$
\begin{aligned}
S_{\mathrm{B} / \mathrm{F}}(\boldsymbol{q}, \boldsymbol{p})= & \mp \frac{1}{(2 \pi \hbar)^{3}} \frac{2 \pi m^{2}}{n \beta q} \frac{1}{1-e^{(\beta / 2 m)\left(2 \sigma(\boldsymbol{q}, \boldsymbol{p}) q-q^{2}\right)}} \\
& \times \sum_{k=1}^{\infty}(-)^{k+1} \frac{(\mp z)^{k}}{k} e^{-(\beta / 2 m) \sigma^{2}(\boldsymbol{q}, \boldsymbol{p})}\left[1-e^{k(\beta / 2 m)\left(2 \sigma(\boldsymbol{q}, \boldsymbol{p}) q-q^{2}\right)}\right] .
\end{aligned}
$$

Considering now in (B1) a geometrical progression of reason $e^{(\beta / 2 m)\left(2 \sigma(q, p) q-q^{2}\right)}$, according to the formula

$$
1-x^{k}=(1-x) \sum_{n=0}^{k-1} x^{n}
$$

Eq. (B1) becomes

$$
S_{\mathrm{B} / \mathrm{F}}(\boldsymbol{q}, \boldsymbol{p})=\frac{1}{(2 \pi \hbar)^{3}} \frac{2 \pi m^{2}}{n \beta q} \sum_{k=1}^{\infty}( \pm)^{k+1} \frac{z^{k}}{k} e^{-k(\beta / 2 m) \sigma^{2}(\boldsymbol{q}, \boldsymbol{p})} \sum_{n=0}^{k-1} e^{n(\beta / 2 m)\left(2 \sigma(\boldsymbol{q}, \boldsymbol{p}) q-q^{2}\right)},
$$

where it is to be noted that the sum over $n$ is due to the presence of the statistical correction 1 $\pm\left\langle n_{k-q}\right\rangle_{\mathrm{B} / \mathrm{F}}$ in (22) and disappears, being replaced by a factor of 1 , if this correction is neglected. It is worthwhile to put into evidence a factor

$$
\frac{1}{(2 \pi \hbar)^{3}} \frac{2 \pi m^{2}}{n \beta q} z e^{-(\beta / 2 m) \sigma^{2}(q, p)}
$$


corresponding to the expression of the dynamic structure factor for a free gas of MaxwellBoltzmann particles, thus obtaining

$$
S_{\mathrm{B} / \mathrm{F}}(\boldsymbol{q}, \boldsymbol{p})=S_{\mathrm{MB}}(\boldsymbol{q}, \boldsymbol{p})\left[1+\sum_{k=1}^{\infty}( \pm)^{k} \frac{z^{k}}{k+1} e^{-k(\beta / 2 m) \sigma^{2}(\boldsymbol{q}, \boldsymbol{p})} \sum_{n=0}^{k} e^{n(\beta / 2 m)\left(2 \sigma(\boldsymbol{q}, \boldsymbol{p}) q-q^{2}\right)}\right] .
$$

In the Brownian limit $\alpha \ll 1$ considered in Sec. IV, neglecting in (30) the contributions of order $\alpha$, this expression goes simply over to

$$
S_{\mathrm{B} / \mathrm{F}}^{\infty}(\boldsymbol{q}, \boldsymbol{p})=S_{\mathrm{MB}}^{\infty}(\boldsymbol{q}, \boldsymbol{p})\left[1+\sum_{k=1}^{\infty}( \pm)^{k} \frac{z^{k}}{k+1} e^{-k(\beta / 8 m) q^{2}} e^{-k(\beta / 2)\left[q^{2} / 2 M+\boldsymbol{q} \cdot \boldsymbol{p} / M\right]} \sum_{n=0}^{k} e^{n \beta\left[q^{2} / 2 M+\boldsymbol{q} \cdot \boldsymbol{p} / M\right]}\right],
$$

where $S_{\mathrm{B} / \mathrm{F}}^{\infty}(\boldsymbol{q}, \boldsymbol{p})$ and $S_{\mathrm{MB}}^{\infty}(\boldsymbol{q}, \boldsymbol{p})$ are given, respectively, by (31) and (32). In terms of $E(\boldsymbol{q}, \boldsymbol{p})$ Eq. (B3) takes the remarkably compact form

$$
S_{\mathrm{B} / \mathrm{F}}^{\infty}(\boldsymbol{q}, E)=S_{\mathrm{MB}}^{\infty}(\boldsymbol{q}, E)\left[1+\sum_{k=1}^{\infty}( \pm)^{k} \frac{z^{k}}{k+1} e^{-k(\beta / 8 m) q^{2}} e^{-k(\beta / 2) E} \sum_{n=0}^{k} e^{n \beta E}\right],
$$

where $S_{\mathrm{B} / \mathrm{F}}^{\infty}(\boldsymbol{q}, E)$ and $S_{\mathrm{MB}}^{\infty}(\boldsymbol{q}, E)$ are given by (33) and (34). We now go one step further noting that the following identity holds:

$$
e^{-k(\beta / 2) E} \sum_{n=0}^{k} e^{n \beta E}=\frac{\sinh [(k+1)(\beta / 2) E]}{\sinh ((\beta / 2) E)},
$$

which can be easily obtained exploiting (B2), so that (B4) becomes

$$
S_{\mathrm{B} / \mathrm{F}}^{\infty}(\boldsymbol{q}, E)=S_{\mathrm{MB}}^{\infty}(\boldsymbol{q}, E) \sum_{k=0}^{\infty}( \pm)^{k} \frac{z^{k}}{k+1} e^{-k(\beta / 8 m) q^{2}} \frac{\sinh [(k+1)(\beta / 2) E]}{\sinh ((\beta / 2) E)} .
$$

Equation (B5) is the most convenient expression in order to consider the limit of small momentum transfer. Exploiting the expansion

$$
\frac{\sinh \left[\frac{1}{2}(k+1) \beta E\right]}{\sinh \left(\frac{1}{2} \beta E\right)}=(k+1)\left[1+\frac{1}{24}(\beta E)^{2}\left(k^{2}+2 k\right)+O\left(E^{4}\right)\right]
$$

and recalling that $E$ is given by (6) we may write (B5) as

$$
\begin{aligned}
S_{\mathrm{B} / \mathrm{F}}^{\infty}(\boldsymbol{q}, E)= & S_{\mathrm{MB}}^{\infty}(\boldsymbol{q}, E)\left[\sum_{k=0}^{\infty}( \pm z)^{k}-\frac{\beta}{8 m} q^{2} \sum_{k=1}^{\infty}( \pm)^{k} k z^{k}\right. \\
& \left.+\frac{1}{12}(\beta E)^{2} \sum_{k=1}^{\infty}( \pm)^{k} k z^{k}+\frac{1}{24}(\beta E)^{2} \sum_{k=1}^{\infty}( \pm)^{k} k^{2} z^{k}+O\left(q^{4}\right)\right] .
\end{aligned}
$$

Recalling (B5) and the explicit expression of $S_{\mathrm{MB}}^{\infty}$ given by (22) one also has

$$
S_{\mathrm{B} / \mathrm{F}}^{\infty}(\boldsymbol{q}, E)= \pm \frac{1}{(2 \pi \hbar)^{3}} \frac{2 \pi m^{2}}{n \beta q} \frac{e^{-(\beta / 2) E}}{\sinh ((\beta / 2) E)} \sum_{k=1}^{\infty}( \pm)^{k} \frac{z^{k}}{k} e^{-k(\beta / 8 m) q^{2}} \sinh \left(k \frac{\beta}{2} E\right)
$$

and, exploiting 22 


$$
\sum_{k=1}^{\infty} \frac{p^{k}}{k} \sinh (k x)=\operatorname{arth}\left[\frac{p \sinh x}{1-p \cosh x}\right]
$$

we obtain the alternative expression

$$
S_{\mathrm{B} / \mathrm{F}}^{\infty}(\boldsymbol{q}, E)= \pm \frac{1}{(2 \pi \hbar)^{3}} \frac{2 \pi m^{2}}{n \beta q} \frac{e^{-(\beta / 2) E}}{\sinh ((\beta / 2) E)} \operatorname{arth}\left[\frac{ \pm z e^{-(\beta / 8 m) q^{2}} \sinh ((\beta / 2) E)}{1 \mp z e^{-(\beta / 8 m) q^{2}} \cosh ((\beta / 2) E)}\right]
$$

equivalent to (31) as can also be directly checked starting from the identity

$$
\operatorname{arth} x=\frac{1}{2} \log \left[\frac{1+x}{1-x}\right] \text {. }
$$

Note that (31) and (B7) in the Fermi case can also be written in the form

$$
S_{\mathrm{F}}^{\infty}(\boldsymbol{q}, E)=-\frac{1}{(2 \pi \hbar)^{3}} \frac{\pi m^{2}}{n \beta q} \frac{e^{-(\beta / 2) E}}{\sinh ((\beta / 2) E)} \log \left[\frac{1-[z /(1+z)]\left(1-e^{-(\beta / 8 m) q^{2}} e^{-(\beta / 2) E}\right)}{1-[z /(1+z)]\left(1-e^{-(\beta / 8 m) q^{2}} e^{+(\beta / 2) E}\right)}\right]
$$

and

$$
S_{\mathrm{F}}^{\infty}(\boldsymbol{q}, E)=-\frac{1}{(2 \pi \hbar)^{3}} \frac{2 \pi m^{2}}{n \beta q} \frac{e^{-(\beta / 2) E}}{\sinh ((\beta / 2) E)} \operatorname{arth}\left[\frac{[z /(1+z)] e^{-(\beta / 8 m) q^{2}} \sinh ((\beta / 2) E)}{[z /(1+z)]\left(1-e^{-(\beta / 8 m) q^{2}} \cosh ((\beta / 2) E)\right)-1}\right],
$$

respectively, which can be useful if one is interested in an expansion for large values of $z$. According to (B8), also for $S_{\mathrm{B} / \mathrm{F}}(\boldsymbol{q}, E)$ given by (23) one has the alternative expression

$$
S_{\mathrm{B} / \mathrm{F}}(\boldsymbol{q}, E)= \pm \frac{1}{(2 \pi \hbar)^{3}} \frac{2 \pi m^{2}}{n \beta q} \frac{e^{-(\beta / 2) E}}{\sinh ((\beta / 2) E)} \operatorname{arth}\left[\frac{ \pm z e^{-(\beta / 8 m) q^{2}} e^{-(\beta / 2)\left(m / q^{2}\right) E^{2}} \sinh ((\beta / 2) E)}{1 \mp z e^{-(\beta / 8 m) q^{2}} e^{-(\beta / 2)\left(m / q^{2}\right) E^{2}} \cosh ((\beta / 2) E)}\right] .
$$

The validity of the detailed balance condition for (B9) according to (12) can immediately be checked observing that both $\sinh x$ and $\operatorname{arth} x$ are odd functions, while $\cosh x$ is an even function.

\footnotetext{
${ }^{1}$ D. Giulini, E. Joos, C. Kiefer, J. Kupsch, I.-O. Stamatescu, and H. D. Zeh, Decoherence and the Appearance of a Classical World in Quantum Theory (Springer, Berlin, 1996).

${ }^{2}$ Proceedings of the 3rd Workshop on Mysteries, Puzzles, and Paradoxes in Quantum Mechanics, edited by R. Bonifacio, B. G. Englert, and D. Vitali, Z. Naturforsch., A: Phys. Sci. 56 (2001).

${ }^{3}$ R. Alicki and K. Lendi, Lecture Notes in Physics (Springer, Berlin, 1987), Vol. 286.

${ }^{4}$ H. Spohn, Rev. Mod. Phys. 52, 569 (1980).

${ }^{5}$ G. Lindblad, Commun. Math. Phys. 48, 119 (1976).

${ }^{6}$ L. Lanz and B. Vacchini, Int. J. Theor. Phys. 36, 67 (1997); Phys. Rev. A 56, 4826 (1997).

${ }^{7}$ B. Vacchini, Phys. Rev. Lett. 84, 1374 (2000).

${ }^{8}$ B. Vacchini, Phys. Rev. E 63, 066115 (2001).

${ }^{9}$ R. F. O’Connell, Phys. Rev. Lett. 87, 028901 (2001); B. Vacchini, ibid. 87, 028902 (2001).

${ }^{10}$ M. Anderson, J. R. Ensher, M. R. Matthews, C. E. Wieman, and E. A. Cornell, Science 269, 198 (1995); B. DeMarco and D. S. Jin, ibid. 285, 1703 (1999).

${ }^{11}$ A. S. Holevo, Rep. Math. Phys. 32, 211 (1993); 33, 95 (1993); Izv. RAN Ser. Mat. 59, 205 (1995); J. Math. Phys. 37, 1812 (1996).

${ }^{12}$ S. W. Lovesey, Theory of Neutron Scattering from Condensed Matter (Clarendon, Oxford, 1984).

${ }^{13}$ R. Haag, N. M. Hugenholtz, and M. Winnink, Commun. Math. Phys. 5, 215 (1967).

${ }^{14}$ L. van Hove, Phys. Rev. 95, 249 (1954).

${ }^{15}$ D. D. Botvich, V. A. Malyshev, and A. D. Manita, Helv. Phys. Acta 64, 1072 (1991).

${ }^{16}$ A. D. Manita, Teor. Mat. Fiz. 89, 366 (1991).

${ }^{17}$ N. G. van Kampen, Stochastic Processes in Physics and Chemistry (North-Holland, Amsterdam, 1981).

${ }^{18}$ A. Barchielli, Nuovo Cimento Soc. Ital. Fis., B 74B, 113 (1983).

${ }^{19}$ G. Lindblad, Rep. Math. Phys. 10, 393 (1976).

${ }^{20}$ A. Isar, A. Sandulescu, and W. Scheid, J. Math. Phys. 34, 3887 (1993); G. Lindblad, ibid. 39, 2763 (1998).
} 
${ }^{21}$ R. K. Pathria, Statistical Mechanics (Butterworth-Heinemann, Oxford, 1996).

${ }^{22}$ I. S. Gradshteyn and I. M. Ryzhik, Table of Integrals, Series, and Products (Academic, Boston, 1994).

${ }^{23}$ A. Săndulescu and H. Scutaru, Ann. Phys. (N.Y.) 173, 277 (1987).

${ }^{24}$ B. Vacchini, Z. Naturforsch., A: Phys. Sci. 56, 230 (2001). 